Phys. Rev. D59, 114027 (1999)

UK/TP 99-01

LPNHE 99-01

SLAC-PUB-8048

hep-ph/9902326

\title{
Radiative Decays, Nonet Symmetry and SU(3) Breaking
}

\author{
M. Benayoun ${ }^{a}$, L. DelBuono ${ }^{a}$, S. Eidelman ${ }^{a, b}$, V. N. Ivanchenko ${ }^{a, b}$, H.B. O'Connell ${ }^{c, d *}$ \\ ${ }^{a}$ LPNHE des Universités Paris VI et VII-IN2P3, Paris, France \\ ${ }^{b}$ Budker Institute of Nuclear Physics, Novosibirsk 630090, Russia \\ ${ }^{c}$ Department of Physics and Astronomy, University of Kentucky, Lexington, KY 40506, USA \\ ${ }^{d}$ Stanford Linear Accelerator Center, Stanford University, Stanford CA 94309, USA
}

(11th February 1999

Revised: 16 March 1999)

\begin{abstract}
We re-examine the problem of simultaneously describing in a consistent way all radiative and leptonic decays of light mesons $(V \rightarrow P \gamma, P \rightarrow V \gamma, P \rightarrow \gamma \gamma$, $\left.V \rightarrow e^{+} e^{-}\right)$. For this purpose, we rely on the Hidden Local Symmetry model in both its anomalous and non-anomalous sectors. We show that the $\mathrm{SU}(3)$ symmetry breaking scheme proposed by Bando, Kugo and Yamawaki, supplemented with nonet symmetry breaking in the pseudoscalar sector, allows one to reach a nice agreement with all data, except for the $K^{* \pm}$ radiative decay. An extension of this breaking pattern allows one to account for this particular decay mode too. Considered together, the whole set of radiative decays provides a pseudoscalar mixing angle $\theta_{P} \simeq-11^{\circ}$ and a value for $\theta_{V}$ which is $\simeq 3^{\circ}$ from that of ideal mixing. We also show that it is impossible, in a practical sense, to disentangle the effects of nonet symmetry breaking and those of glue inside the $\eta^{\prime}$, using only light meson decays.
\end{abstract}

*Work supported by Department of Energy contract DE-AC03-76SF00515. 
When in doubt, tell the truth ...

Mark Twain

\section{INTRODUCTION.}

Defining an accurate framework in which the study of all radiative decays of light flavor mesons can be performed successfully is a long standing question. There are a few kinds of different models which have been proposed so far. The most popular modelling is in terms of magnetic moments of quarks [1.2]. It includes, to some extent, $\mathrm{SU}(3)$ breaking effects by having a magnetic moment for the $s$ quark, slightly different to that of the $d$ quark. It also depends on overlap integrals which are hard to estimate theoretically, and are thus arbitrarily chosen equal [3]. This, at least, allows one to have a reasonable number of free parameters. Another traditional approach is to use $\mathrm{SU}(3)$ relations among coupling constants [ [4]. However, the assumption of exact SU(3) symmetry still yields reasonable descriptions of radiative decays [5], though the success is never complete.

Recently, several models including SU(3) symmetry breaking effects have been proposed [6 8], motivated in part by effective Lagrangian approaches to the interactions of vector mesons [9, 10], sometimes with additional SU(2) symmetry breaking effects [7]. Including SU(3) symmetry breaking as per Bando, Kugo and Yamawaki (hereafter referred to as BKY) [11,12], these models give a special role to all decay constants $\left(f_{K}, f_{\eta}, f_{\eta^{\prime}}\right)$ in the breaking procedure; some additional corrections are also allowed. More recently, a new kind of model has been proposed [13], where additional symmetry breaking effects are introduced by means of the (measured) leptonic decay constants of vector mesons.

The study of radiative decays of light flavor mesons is also connected with the long standing problem of $\eta / \eta^{\prime}$ mixing [5, 8, 14 [17] and to its possible association with a glue content [13, 18]. The two-photon decay of the $\eta\left(\eta^{\prime}\right)$ is also connected with the problem of anomalies 18 21. Recent developments seem to advocate a more complicated $\eta / \eta^{\prime}$ mixing scheme [22.23], which has received support from some phenomenological analyses [24,25]. Another approach in the same vein has been proposed quite recently by Escribano and Frère [26], where the pseudoscalar mixing angle $\theta_{P}$ is taken to be mass dependent. Practically, this leads to two different mixing angles $\theta_{\eta}=\theta_{P}\left(m_{\eta}^{2}\right)$ and $\theta_{\eta^{\prime}}=\theta_{P}\left(m_{\eta^{\prime}}^{2}\right)$ in order to express the physical states $|\eta\rangle$ and $\left|\eta^{\prime}\right\rangle$ in terms of the octet and singlet pseudoscalar fields. As a consequence of this assumption, however, the physical states $\mid \eta>$ and $\mid \eta^{\prime}>$ are no longer orthogonal to each other. Similar discussions can be found for $\pi^{0}-\eta$ mixing [27] and $\rho-\omega$ mixing [28], where the latter is a necessary result of current conservation [29].

Among the models sketched so far, only the O'Donnell model [4] introduces, quite naturally, the effect of nonet symmetry breaking, simply by its being $\mathrm{SU}(3)$ (but not $\mathrm{U}(3)$ ) symmetric in both the pseudoscalar and vector sectors. As phenomenology tends to indicate the relevance of deviations from nonet symmetry, it looks interesting to keep this possibility, in addition to conventional $\mathrm{SU}(3)$ breaking effects.

The purpose of the present paper is to propose a new model based on the successful approach of hidden local symmetry [9], and particularly its anomalous sector [10]. As we know that effects of SU(3) symmetry breaking are clearly observed in the data [5], they have surely to be introduced. We do it following the BKY mechanism [11]. This approach 
reveals an interesting pattern for the $s-q$ breaking ( $q$ or $q^{\prime}$ stand for $u$ or $d$ quarks). Indeed, decays involving a $\left(q \overline{q^{\prime}}\right)$ pseudoscalar meson have unchanged coupling constants (they depend directly on $\left.f_{\pi}\right)$, while decays involving a $(s \bar{q})$ or a $(\bar{s} q)$ pseudoscalar meson are renormalized by $f_{\pi} / f_{K}$, and the $(s \bar{s})$ part (only) of neutral meson couplings is corrected by $\left(f_{\pi} / f_{K}\right)^{2}$, without any change for the additional $(q \bar{q})$ parts.

This is automatically performed by a field renormalization [11,12, which must be propagated [12] into the interaction (non-anomalous and anomalous) Lagrangians. This breaking scheme has the virtue of being both simple and successfully predicting the magnitude and the functional form of the breaking effects. In order to probe various kinds of effects, we supplement the BKY breaking by another one introduced by Bramon, Grau and Pancheri [6] (hereafter referred to as BGP) which acts directly on the anomalous Lagrangian of Ref. [10]. Finally, we introduce there the possibility of breaking the nonet symmetry in the pseudoscalar sector.

The net result of this breaking pattern is threefold. Firstly, to explicitly construct a Lagrangian model which gives the $\mathrm{SU}(3)$ model of Ref. [4] for $V P \gamma$ transitions. Secondly to perform consistently the BKY and BGP breakings, and thirdly to test against data each of the elementary breaking schemes (BKY, BGP and nonet symmetry) separately. Finally, we will also examine the effects of a glue component coupled to the $\eta$ and $\eta^{\prime}$ mesons.

On another hand, using standard Feynman rules, this model also allows us to reconstruct the amplitudes for the anomalous decays $\eta / \eta^{\prime} \rightarrow \gamma \gamma$. It so happens that it provides the traditional mixing scheme with only one mixing angle $\theta_{P}$ and with, additionally, expressions for the singlet and octet decay constants $f_{1}$ and $f_{8}$ in terms of $f_{\pi}, f_{K}$ and the nonet symmetry breaking parameter (named $x$ ). We thus have an access to testing whether radiative decays of type $V P \gamma$ give support to this traditional scheme [15 [17,20,21], or if there is evidence for its failure, in connection with the recent modelling involving two mixing angles [22 26].

The paper is organized as follows. In Section [I], we sketch the present status of experimental data. In Section III we summarize the standard exact SU(3) model of O'Donnell for radiative decays and examine how the interplay of an additional singlet can be performed. Section $[\mathrm{IV}$ is devoted to a brief reminder of the main tools : the HLS model, its anomalous sector and the SU(3) symmetry breaking schemes. It is here (Subsection IVD) that we exhibit the Lagrangian model we use in order to describe the radiative and leptonic decays of light flavor mesons. We discuss here, and in Section $\nabla$, the way nonet symmetry breaking is implemented and the connection between the symmetry breaking parameters and the usual singlet and octet decay constants $f_{1}$ and $f_{8}$. In Section $\nabla \mathbb{1}$, we discuss the results of the fit using the proposed model. The analysis of nonet symmetry breaking versus glue component in the $\eta$ and $\eta^{\prime}$ mesons is discussed in Section VII. Problems connected with the Primakoff measurement of $\eta$ decay width are shortly discussed in Section VIII. Predictions for branching fractions are presented in Sections $[\mathrm{IX}$ and $\mathrm{X}$. Section $\mathrm{XI}$ deals with a special treatment for including the $K^{* \pm}$ radiative decay mode inside the set of partial widths submitted to fit. Section XII is devoted to conclusions. For ease of reading, most of the numerical results are gathered in Tables and most formulae are given in the Appendix. 


\section{THE DATA}

Recently there have been a few improvements of the data we analyze. Most of them have already been listed in Review of Particle Properties [30]. This concerns some decay modes like $\rho^{0} \rightarrow \eta \gamma$ and $\rho^{0} \rightarrow \pi^{0} \gamma$ which were previously somewhat overestimated [31. Results have also been improved for the mode $\omega \rightarrow \eta \gamma$ and new results have increased the quality of the information on $\phi$ radiative decay to $\eta[32,33]$. Moreover, the decay mode $\phi \rightarrow \eta^{\prime} \gamma$ has been measured by the two detectors mounted on the VEPP-2M collider. The result of the CMD2 collaboration [34] is the present reference [30 and corresponds to a branching ratio of $\left(1.2_{-0.5}^{+0.7}\right) \times 10^{-4}$. More recently, the SND collaboration has reported [35] a slightly smaller (but consistent within errors) branching ratio of $\left(6.7_{-2.9}^{+3.4}\right) \times 10^{-5}$.

Therefore, we have at our disposal the full set of radiative decays of light mesons. One might, however, expect potential difficulties with some particular data. The first is the disagreement between the Primakoff effect measurement of $\Gamma(\eta \rightarrow \gamma \gamma)$, and the results which have been obtained from six $e^{+} e^{-}$experiments (see Ref. [30] for references). One should note that the results provided by all $e^{+} e^{-}$experiments are statistically consistent with each other (mean value : $0.514 \pm 0.026 \mathrm{keV})$ whereas the Primakoff effect measurement $(0.324 \pm$ $0.046 \mathrm{keV}$ ) is statistically inconsistent with $e^{+} e^{-}$experiments by more than $3 \sigma$. Therefore, it could be considered unreliable to mix the results from $e^{+} e^{-}$data with those from the Primakoff effect. Hence, in the following, we use the mean value of the $e^{+} e^{-}$measurements $(0.514 \pm 0.026 \mathrm{keV})$, which seems more reliable, due to the number of experiments. We shall, however, somehow discuss the physical consequences of the Primakoff measurement $(0.324 \pm 0.046 \mathrm{keV})$.

The second (potential) problem is the self-consistency of the measured values for the partial widths $K^{* \pm} \rightarrow K^{ \pm} \gamma$ and $K^{* 0} \rightarrow K^{0} \gamma$ which have been recognized hard to reconcile with each other by several authors. For instance the model of exact $\mathrm{SU}(3)$ symmetry [4] provides [5] a ratio $\Gamma\left(K^{* 0} \rightarrow K^{0} \gamma\right) / \Gamma\left(K^{* \pm} \rightarrow K^{ \pm} \gamma\right)=4$, as the (SU(3) broken symmetry) model of Ref. [13], while the reported data [30] rather give $2.2 \pm 0.3$. On the other hand, the model using quark magnetic moments predicts [36] a ratio of 1.65. In these last two cases, the reported value for $\Gamma\left(K^{* 0} \rightarrow K^{0} \gamma\right)$ is well reproduced, while the reported information [30] for $\Gamma\left(K^{* \pm} \rightarrow K^{ \pm} \gamma\right)$ is twice larger compared to the prediction of Ref. [13] or $50 \%$ smaller compared to the prediction of Ref. [36]. It is hard to determine the source of this discrepancy, which could conceivably be either due to systematic effects in the measurements or simply reflect the need for a refined modelling.

\section{AN EXACT SU(3) SYMMETRY FRAMEWORK}

The formalism which describes the decays $V \rightarrow P \gamma$ and $P \rightarrow V \gamma$ within an exact $\mathrm{SU}(3)$ symmetry framework has been given by P. O'Donnell in Ref. [4. The corresponding decay amplitudes can be quite generally円 written as

$$
T=g_{V P \gamma} \epsilon_{\mu \nu \rho \sigma} k^{\mu} q^{\nu} \varepsilon^{\rho}(V) \varepsilon^{\sigma}(\gamma)
$$

\footnotetext{
1 That is, independently of any specific Lagrangian.
} 
using obvious notations. The coupling strengths $g_{V P \gamma}$ between physical vector and pseudoscalar mesons in radiative decays are expressed in terms of two angles $\left(\theta_{V}\right.$ and $\left.\theta_{P}\right)$ which describe the mixtures of singlet and octet components, and of three coupling constants $\left(g_{V_{8} P_{8} \gamma}, g_{V_{1} P_{8} \gamma}\right.$ and $\left.g_{V_{8} P_{1} \gamma}\right)$; indeed, assuming that the photon behaves like an SU(3) octet cancels out the possible coupling $g_{V_{1} P_{1} \gamma}$. We do not reproduce here the expressions for the $g_{V P \gamma}$ in terms of the elementary couplings $g_{V_{i} P_{j} \gamma}$ and the mixing angles; they can be found in Ref. [4] and in Appendix A7 of Ref. [5], where a misprint has been corrected. These formulae use mixing angles describing deviations from ideal mixing, introduced long ago in Ref. [37], rather than the more customary mixing angles relative to octet and singlet components. The relevance of this angle definition has recently been rediscovered [24.25 in connection with the $\eta / \eta^{\prime}$ mixing problem.

At this point, it should be noted that exact $\mathrm{SU}(3)$ symmetry is not in conflict with releasing the condition of nonet symmetry (which corresponds to the stronger $\mathrm{U}(3)$ symmetry) usually stated in effective Lagrangian models for both the vector and pseudoscalar meson sectors [8 10, 38, 39. Moreover, the O'Donnell formulation also treats the mixing angles as free parameters to be determined?.

Then, assuming the existence of only one singlet for each of the vector and pseudoscalar meson sectors, exact SU(3) symmetry gives a description which depends generally on five parameters. Reducing the number of free parameters requires additional symmetries. In most traditional approaches to vector meson physics, nonet symmetry in the vector sector is assumed [8 10,38, 39] once the vector meson field matrix has been written with ideal $\omega$ and $\phi$ fields. A recent phenomenological study of light meson radiative decays [5] has concluded that all existing data highly favor nonet symmetry in the vector meson sector. Practically, this means that one can limit oneself to only two independent couplings: $G=g_{V_{8} P_{8} \gamma}=g_{V_{1} P_{8} \gamma}$ and $G_{1}^{\prime}=g_{V_{8} P_{1} \gamma}$; if we define $x$ by $G_{1}^{\prime}=x G$, the result of the most likely fits to the data in Ref. [5] (the so-called "internal fit" and "model M1"), tells that $x \simeq 0.9$, with a (statistical) error making $x$ fully inconsistent with 1 . This would imply that nonet symmetry is significantly broken in the pseudoscalar sector. Whether this value for $x$ is a fundamental property of radiative decays, or an effective way to account for (ignored) SU(3) symmetry breaking effects, is still an open question and partly motivates the present paper.

The question of whether nonet symmetry in the pseudoscalar sector is fulfilled is intimately related to the $\mathrm{U}(1)_{A}$ anomaly and recent accounts of this problem can be found in Refs. 40,22,23. At large $N_{c}$, the nine basic pseudoscalar mesons should form a nonet of degenerate Goldstone bosons, and then one could expect that the parameter $x$ defined above should tend to 1 . However, the precise value of $x$ should also be related to the scale dependence of the singlet coupling constant 18,22,23]. Therefore, from a phenomenological point of view, it looks wise to let $x$ vary and examine the consequences of assigning it specific values (including 1).

Moreover, recent theoretical developments tend to advocate that the singlet sector of pseudoscalar mesons could well be not saturated [13, 18, 22, 40] by the standard singlet $v_{1}=$ $(u \bar{u}+d \bar{d}+s \bar{s}) / \sqrt{3}$ only. One (or more) of the glueballs predicted by QCD could play a

\footnotetext{
${ }^{2}$ The relation of mixing angles to meson masses, and thus to some $\mathrm{SU}(3)$ breaking effects, is ignored, allowing one to compare fit values with theoretical expectations.
} 
non-negligible role. Let us denote this additional state $v_{1}^{\prime}=g g$, exhibiting its possible connection with glue. Actually such a state could be a compound of several SU(3) singlets, like several glueballs with, even, a possible admixture of $c \bar{c}$, as recently advocated in order to account for the unexpectedly high rate of $B \rightarrow \eta^{\prime} K$ observed at CLEO [41] (see Refs. 42, 24] and references quoted therein). The possibility that a part of the broad structure seen in radiative $J / \psi$ decays and presently named [30] $\eta(1440)$ could be a nearly pure glueball is still considered [43] (see Ref. [44] and the minireview in Ref. [30]).

Then, it is meaningful to allow for the mixing of $v_{8}=(u \bar{u}+d \bar{d}-2 s \bar{s}) / \sqrt{6}$ with both singlet states already referred to as $v_{1}$ and $v_{1}^{\prime}$. This follows the proposal in Ref. [13]. We are not actually very dependent on an assumption about the precise content of $v_{1}^{\prime}$, except that it is supposed to be orthogonal to $v_{1}$.

It is suitable, for later use, to choose a parametrization of the mixing of $\left(v_{8}, v_{1}, v_{1}^{\prime}\right)$ into physical pseudoscalar meson states denoted $\left(\eta, \eta^{\prime}, \eta^{\prime \prime}\right)$. Using the symbol $\eta^{\prime \prime}$ for the third partner of the doublet $\left(\eta, \eta^{\prime}\right)$ simply means that we don't care to identify it, as we do not presently plan to describe its coupling to vector mesons and photons and, more generally, its physics. Any general parametrization of an orthogonal rotation matrix depends a priori on 3 angles. One could for instance choose to express it in terms of the usual Euler angles, however, an appropriate parametrization of this transform is represented by the CabibboKobayashi-Maskawa matrix (with the complex phase factor $\delta$ removed)

$\left[\begin{array}{c}\eta \\ \eta^{\prime} \\ \eta^{\prime \prime}\end{array}\right]=\left[\begin{array}{lll}\cos \theta \cos \beta & \sin \beta \\ \sin \theta \cos \gamma-\cos \theta \sin \beta \sin \gamma & \cos \theta \cos \gamma+\sin \theta \sin \beta \sin \gamma & \cos \beta \sin \gamma \\ -\sin \theta \sin \gamma-\cos \theta \sin \beta \cos \gamma & -\cos \theta \sin \gamma+\sin \theta \sin \beta \cos \gamma & \cos \beta \cos \gamma\end{array}\right]\left[\begin{array}{c}v_{8} \\ v_{1} \\ v_{1}^{\prime}\end{array}\right]$

Indeed, the vanishing of $\beta$ and $\gamma$ gives smoothly the usual mixing pattern of the $\left(\eta, \eta^{\prime}\right)$ doublet (with $\theta \equiv \theta_{P}$ ) and the decoupling of the additional singlet (which will be frequently - and abusively -named glue). Setting $\beta=0$ cancels out glue inside $\eta$ only, while $\gamma=0$ removes any glue inside the $\eta^{\prime}$ only.

\section{THE HLS MODEL AND SU(3) SYMMETRY BREAKING}

In order to break $\mathrm{SU}(3)$ symmetry relations among coupling constants, it is convenient to refer to a well defined framework, in which clear meaning can be ascribed to each parameter, particularly to parameters which define the breaking procedure itself. In this way, fit parameters and values can be physically interpreted, which is a missing information noticed [13] for the parameters $g, g_{1}$ and $g_{1}^{\prime}$ of Ref. [5].

\footnotetext{
${ }^{3}$ These parameters have already been renamed with the corresponding capital letters in order to avoid ambiguities with other meanings for the same symbols in use in the field of effective Lagrangian models.
} 


\section{A. The Unbroken HLS Model}

We will partly work within the framework of the hidden local symmetry model (referred to in the following as HLS). A full account of this can be found in Refs. [9, 11] and, for what concerns the anomalous sector, also in Ref. [10]. Brief accounts can be found in Refs. [6, 0, 12, 38] and will not be repeated here. However, in order to fix - and modify - notations, a few points have to be recalled. The HLS Lagrangian can be written $\mathcal{L}_{\mathrm{HLS}}=\mathcal{L}_{A}+a \mathcal{L}_{V}$ where

$$
\begin{aligned}
& \mathcal{L}_{A}=-\frac{f_{P}^{2}}{4} \operatorname{Tr}\left[D_{\mu} \xi_{L} \xi_{L}^{\dagger}-D_{\mu} \xi_{R} \xi_{R}^{\dagger}\right]^{2} \\
& \mathcal{L}_{V}=-\frac{f_{P}^{2}}{4} \operatorname{Tr}\left[D_{\mu} \xi_{L} \xi_{L}^{\dagger}+D_{\mu} \xi_{R} \xi_{R}^{\dagger}\right]^{2}
\end{aligned}
$$

and $a$ is a parameter which is not fixed by the theory. Setting $a=2$ allows one to recover the usual expression for vector meson dominance (VMD) 9] (for a review of VMD see, for example Ref. 45]). However, some experimental evidence 46,47 indicates that $a$ is slightly (but significantly) greaterf than 2. For this reason, we prefer to keep $a$ free. After removal of a scalar field matrix, we have

$$
\xi_{L}^{\dagger}=\xi_{R}=\xi=e^{i P(x) / f_{P}}
$$

where $P$ is the pseudoscalar field matrix and $f_{P}$ is usually identified with the pion decay constant $f_{\pi}=92.42 \mathrm{MeV}$ [30], at least for phenomenological purposes. The HLS Lagrangian above is gauged for both electromagnetism and the hidden local symmetry through the covariant derivative

$$
D_{\mu} \xi_{L, R}=\partial_{\mu} \xi_{L, R}-i g V_{\mu} \xi_{L, R}+i e \xi_{L, R} A_{\mu} Q
$$

where $A_{\mu}$ is the electromagnetic field and $Q=\operatorname{diag}(2 / 3,-1 / 3,-1 / 3)$ is the quark charge matrix. $V$ is the vector meson field matrix

$$
V=\frac{1}{\sqrt{2}}\left(\begin{array}{ccc}
\left(\rho^{0}+\omega^{I}\right) / \sqrt{2} & \rho^{+} & K^{*+} \\
\rho^{-} & \left(-\rho^{0}+\omega^{I}\right) / \sqrt{2} & K^{* 0} \\
K^{*-} & \bar{K}^{* 0} & -\phi^{I}
\end{array}\right) .
$$

Note the superscript $I$ for the $\omega$ and $\phi$ fields, which reminds that these fields correspond to ideal mixing; note also the sign in front of $\phi^{I}$ which defines $\phi^{I} \equiv-s \bar{s}$. There are some reason to think that there is some admixture of non-strange quarks inside the observed $\phi$ meson (for instance, in order to account for the large branching fraction $\phi \rightarrow \pi^{+} \pi^{-} \pi^{0}$ ). This is quite traditionally treated by stating that the observed doublet $(\omega, \phi)$ (denoted without superscripts) is obtained by rotating the doublet $\left(\omega^{I}, \phi^{I}\right)$ in the following way (without any additional change of sign, if we define the ideal $\phi$ as we did)

\footnotetext{
${ }^{4}$ Ref. 48 reached a similar conclusion when analyzing resonance parameters in a model close to HLS.
} 


$$
\left(\begin{array}{l}
\omega \\
\phi
\end{array}\right)\left(\begin{array}{cc}
\cos \delta_{V} & \sin \delta_{V} \\
-\sin \delta_{V} & \cos \delta_{V}
\end{array}\right)\left(\begin{array}{c}
\omega^{I} \\
\phi^{I}
\end{array}\right)
$$

Thus ideal mixing corresponds to $\delta_{V}=0$; correspondingly, the mixing angle of the $\omega, \phi$ system with respect to their octet and singlet components is

$$
\theta_{V}=\theta_{0}+\delta_{V} \quad, \quad \tan \theta_{0}=1 / \sqrt{2} .
$$

In terms of the singlet and octet fields, the pseudoscalar field matrix can be written

$$
P=\frac{1}{\sqrt{2}}\left(\begin{array}{ccc}
\frac{1}{\sqrt{2}} \pi^{0}+\frac{1}{\sqrt{6}} \pi_{8}+\frac{1}{\sqrt{3}} \eta_{0} & \pi^{+} & K^{+} \\
\pi^{-} & -\frac{1}{\sqrt{2}} \pi^{0}+\frac{1}{\sqrt{6}} \pi_{8}+\frac{1}{\sqrt{3}} \eta_{0} & K^{0} \\
K^{-} & K^{0} & -\sqrt{\frac{2}{3}} \pi_{8}+\frac{1}{\sqrt{3}} \eta_{0}
\end{array}\right),
$$

where an explicit use of nonet symmetry fixes the relative weights of the isoscalar terms. In a way analogous to the vector meson case, the connection of $\eta_{0}$ and $\pi_{8}$ with the observable $\eta$ and $\eta^{\prime}$ is defined by a rotation (i.e. defined by a single angle

$$
\left(\begin{array}{c}
\eta \\
\eta^{\prime}
\end{array}\right)\left(\begin{array}{cc}
\cos \theta_{P} & -\sin \theta_{P} \\
\sin \theta_{P} & \cos \theta_{P}
\end{array}\right)\left(\begin{array}{l}
\pi_{8} \\
\eta_{0}
\end{array}\right)
$$

We define also the relation between $\eta$ and $\eta^{\prime}$ and the non-strange $\left(\simeq \omega^{I}\right)$ and strange $\left(\simeq \phi^{I}\right)$ pseudoscalar field combinations by relations analogous to Eq. (7) with a phase $\delta_{P}$ analogous to $\delta_{V}$ in Eq. (8).

The unbroken (nonet symmetric) HLS Lagrangian is given explicitly in Ref. [12]. In order to stay consistent with the convention we have adopted above concerning the $\phi^{I}$ field, all coefficients of terms involving a single $\phi^{I}$ field in Ref. [12] must change their sign.

\section{B. Nonet Symmetry Breaking}

As recalled in Section [II, there is no experimental or phenomenological reason to suspect any failure of nonet (i.e. $\mathrm{U}(3)$ ) symmetry in the vector meson sector. However, we have also recalled that there are phenomenological and theoretical reasons to suspect that nonet symmetry in the pseudoscalar sector might not hold exactly. In order to test this, it is wise to allow for its possible violation in a way which permits a smooth connection from broken to unbroken nonet symmetry. Having to introduce only one singlet combination (denoted $v_{1}$ in Section [II]) might also be questioned [13,18] as commented above.

\footnotetext{
${ }^{5}$ Ref. [26] prefers changing this standard definition by introducing two mixing angles $\theta_{\eta}$ and $\theta_{\eta^{\prime}}$ instead of $\theta_{P}$; we shall briefly comment on this point later on.

${ }^{6}$ The states corresponding to $\omega^{I}$ and $\phi^{I}$ for the pseudoscalar isoscalar mesons are named respectively $\eta_{q}$ and $\eta_{s}$ in Refs. 24, 25.
} 
The most straightforward way to include deviations from nonet symmetry is to do it from the very beginning in the HLS non-anomalous Lagrangian by making the replacement $\eta_{0} \longrightarrow x \eta_{0}$. As can be seen from Eq. (A1) in Ref. [12], this does not influence the interaction terms (which do not contain any interaction with the singlet $\eta_{0}$ ), but only the (omitted) kinetic energy term $\partial \pi_{8} \partial \pi_{8}+\partial \eta_{0} \partial \eta_{0}$ which then becomes $\partial \pi_{8} \partial \pi_{8}+x^{2} \partial \eta_{0} \partial \eta_{0}$. This could imply a redefinition of the singlet decay constant as $f^{\prime}=x f_{P}$ along the lines of Refs. [6.49]. However, if we replace the $\pi^{8}$ and $\eta_{0}$ fields by the physical $\eta$ and $\eta^{\prime}$, this anyway produces a term proportional to $\partial \eta \partial \eta^{\prime}$ which goes to zero when $x \rightarrow 1$. Moreover, as the mass associated with the singlet is a priori different from that of the octet (see for instance Refs. [50, 22]), the same rotation generates a term of the form $m^{2} \eta \eta^{\prime}$ proportional to some mass squared (denoted $m^{2}$ ). When assuming $x=1$, the diagonalization of this mass matrix gives a definition of the mixing angle in terms of physical meson masses. If $x \neq 1$, the situation is not completely clear.

Anyway, the problem we meet with $\eta$ and $\eta^{\prime}$ is tightly connected with the difficult $U(1)_{A}$ anomaly problem, and the present work cannot pretend to solve it. As our main concern is to build a Lagrangian model which allows one to go beyond elementary SU(3) symmetry in describing radiative decays, we will simply assume that this part of the kinetic energy term can be suitably arranged, even when breaking nonet symmetry.

From the point of view of phenomenology, we could just as well introduce this specific breaking directly in the interaction Lagrangian of relevance for our purpose (see below). When having to study possible effects of an additional singlet (named $v_{1}^{\prime}$ above) in the radiative decays involving $\eta$ and $\eta^{\prime}$, we will also assume that the corresponding kinetic energy term can be safely introduced[?

\section{SU(3) Breaking Mechanisms of the HLS Model}

Basically, the SU(3) breaking scheme we use has been introduced by Bando, Kugo and Yamawaki [11] (referred to as BKY) and has given rise to a few variants [6.12], as well as to extension to SU(2) breaking [7]. We refer the reader to Refs. [6, 11,12] for detailed analyses of the properties of known variants of the BKY breaking scheme.

In the following, we use basic consequences common to the original BKY mechanism [11], its hermitized variant and the so-called new scheme, both discussed in Ref. [12]. In these cases, $\mathrm{SU}(3)$ symmetry breaking defines a renormalized pseudoscalar field matrix $P^{\prime}$ in terms of the bare one $P$ given above by

$$
P^{\prime}=X_{A}^{1 / 2} P X_{A}^{1 / 2}
$$

where the breaking matrix $X_{A}$ writes $\operatorname{diag}\left(1,1,1+c_{A}\right)$ and we have [11,12]

${ }^{7}$ At the computational level, it could be done by introducing a $g$ entry in the meson field matrices which thus become $4 \times 4$, filled with zeros, except for the $g g$ location of the pseudoscalar matrix which is filled with this additional term, and extending the $Q$ matrix by a fourth diagonal entry with zero charge. 


$$
\ell_{A} \equiv 1+c_{A}=\left(\frac{f_{K}}{f_{\pi}}\right)^{2}=1.495 \pm 0.030 \quad,
$$

It should be noted [12], that the field renormalization (Eq. (11)) is requested in order to recover the charge normalization condition, $F_{K^{+}}(0)=1$, expected for the kaon form factor $F_{K^{+}}(s)$, even in presence of $\mathrm{SU}(3)$ breaking. The numerical value just given is deduced from the experimental information quoted in Ref. [30].

Concerning vector mesons, apart from changing the couplings of $K^{*}$ and $\phi$ mesons to pseudoscalar pairs, SU(3) breaking modifies the vector meson mass terms and their coupling to the electromagnetic field in the following way

$$
\begin{aligned}
\mathcal{L}=\cdots+ & \frac{1}{2} a f_{\pi}^{2} g^{2}\left[\left(\rho^{0}\right)^{2}+\left(\omega^{I}\right)^{2}+\ell_{V}\left(\phi^{I}\right)^{2}\right] \\
& -a e f_{\pi}^{2} g\left[\rho^{0}+\frac{1}{3} \omega^{I}+\ell_{V} \frac{\sqrt{2}}{3} \phi^{I}\right] . A+\cdots
\end{aligned}
$$

in terms of ideally mixed states. We have defined $\ell_{V} \equiv\left(1+c_{V}\right)^{2}$. Using Eq. (7), we can reexpress the HLS Lagrangian in terms of physical field combinations. The Lagrangian piece given in Eq. (13) thus becomes

$$
\begin{aligned}
\mathcal{L}= & a f_{\pi}^{2} g^{2}\left[\left(\rho^{0}\right)^{2}+\left(\cos ^{2} \delta_{V}+\ell_{V} \sin ^{2} \delta_{V}\right) \omega^{2}+\left(\sin ^{2} \delta_{V}+\ell_{V} \cos ^{2} \delta_{V}\right) \phi^{2}\right]+a f_{\pi}^{2} g^{2} \ell_{V} \omega \cdot \phi \\
& -a e f_{\pi}^{2} g\left[\rho^{0}+\frac{1}{3}\left(\cos \delta_{V}+\ell_{V} \sqrt{2} \sin \delta_{V}\right) \omega-\frac{1}{3}\left(\sin \delta_{V}-\ell_{V} \sqrt{2} \cos \delta_{V}\right) \phi\right] . A
\end{aligned}
$$

The coefficients affecting the $\rho, \omega$ and $\phi$ fields in the last term, are commonly denoted $-e f_{\rho \gamma},-e f_{\omega \gamma}$ and $-e f_{\phi \gamma}$. They are estimated from the vector meson decay widths to $e^{+} e^{-}$ by

$$
\Gamma\left(V \rightarrow e^{+} e^{-}\right)=\frac{4 \pi \alpha_{e m}^{2}}{3 m_{V}^{3}}\left|f_{V \gamma}\right|^{2}
$$

(for a recent discussion of the determination of the "leptonic widths" of vector mesons, see Ref. [51]). Following recent evidence [46,47] that a could be somewhat different from 2, it should be noted that $a$ influences the description of these decays.

One should note the occurrence of a direct transition term $\omega . \phi$, generated by the rotation $\delta_{V}$, which vanishes when the masses of the $\omega$ and $\phi$ mesons become equal (no SU(3) breaking). This term plays an important role when computing some matrix elements, as will be illustrated below.

Some connection between the value of vector meson masses and the value of $c_{V}$ could be inferred [6, 11, 12]; however, given the possible definition dependence of the measured values for vector meson masses [39, it cannot be considered secure a priori. In most variants of the BKY breaking mechanism, $c_{A}$ and $c_{V}$ remain unrelated, except for one of these variants 112 where the condition $c_{A}=a c_{V}$ looks desirable. The explicit expressions for the $\mathrm{SU}(3)$ broken HLS Lagrangians using several breaking schemes can be found in Ref. [12].

\footnotetext{
${ }^{8}$ Notice the square in the definition of $\ell_{V}$.
} 


\section{A Phenomenological Lagrangian for Radiative Decays}

Following Ref. [10] (referred to hereafter as FKTUY), the anomalous, U(3) symmetric, Lagrangian describing $P V V$ interactions and, together with Eqs. (13) and (14), $P V \gamma$ and $P \gamma \gamma$ transitions is given by

$$
\mathcal{L}=-\frac{3 g^{2}}{4 \pi^{2} f_{\pi}} \epsilon^{\mu \nu \rho \sigma} \operatorname{Tr}\left[\partial_{\mu} V_{\nu} \partial_{\rho} V_{\sigma} P\right]
$$

where the coefficient is such that the $\pi^{0} \rightarrow \gamma \gamma$ amplitude has the expression expected from the triangle anomaly 1921$]\left(\alpha / \pi f_{\pi}\right)$. The $V$ and $P$ matrices have been defined above in terms of singlet and octet fields (pseudoscalars mesons) or of ideally mixed states (vector mesons).

One should mention that using the $V$ and $P$ matrices defined above, allows one to recover the $V P \gamma$ couplings of Ref. [4] in the case of nonet symmetry in vector and pseudoscalar sectors. The most general form could be obtained by breaking quite generally the $\mathrm{U}(3)$ symmetry. This can be achieved by weighting the singlet part of the matrix $V$ by a parameter $y$ and that of $P$ by another one, $x$. Therefore, supplemented in this way, Eq. (16) is an appropriate Lagrangian representation for the amplitudes of Eq. (1), in which breaking procedures can be clearly implemented.

The field renormalization following the BKY SU(3) breaking should be propagated down to the FKTUY Lagrangian using Eq. (11)

$$
\mathcal{L}=-\frac{3 g^{2}}{4 \pi^{2} f_{\pi}} \epsilon^{\mu \nu \rho \sigma} \operatorname{Tr}\left[\partial_{\mu} V_{\nu} \partial_{\rho} V_{\sigma} X_{A}^{-1 / 2} P^{\prime} X_{A}^{-1 / 2}\right] .
$$

Then, the VVP Lagrangian is changed in a definite way by the symmetry breaking parameter $\ell_{A}$ defined above (see Eq. (12)) and supposed to have a well understood numerical value (practically 1.5). We also allow for slightly more freedom by introducing the possibility of another SU(3) breaking mechanism, specific to the anomalous Lagrangian, initially proposed by Bramon, Grau and Pancheri [6] (referred to as BGP). This changes Eq. (17) to

$$
\mathcal{L}=-\frac{3 g^{2}}{4 \pi^{2} f_{\pi}} \epsilon^{\mu \nu \rho \sigma} \operatorname{Tr}\left[\partial_{\mu} V_{\nu} X_{W} \partial_{\rho} V_{\sigma} X_{A}^{-1 / 2} P^{\prime} X_{A}^{-1 / 2}\right]
$$

where $X_{W}=\operatorname{diag}\left(1,1,1+c_{W}\right)$ is a new symmetry breaking matrix depending on a new (free) parameter $\ell_{W}=1+c_{W}$. In Section $\nabla$ we shall provide this parameter with a physical meaning analogous to the relation between $\ell_{A}$ and $f_{K} / f_{\pi}$.

The renormalized $P^{\prime}$ matrix is given in Eq. (9), except that we allow for nonet symmetry breaking by making the replacement $\eta_{0} \longrightarrow x \eta_{0}$. Therefore, the Lagrangian which we will first use to describe radiative decays depends a priori on the 2 (free) mixing angles and 3 breaking parameters: $\ell_{A}$ (expected to be of the order 1.5), $\ell_{W}$, and $x$ (expected [5] to be of the order 0.9 , except if explicit $\mathrm{SU}(3)$ breaking is sufficient to fully restore nonet symmetry). The precise values of the HLS parameter $a$ and of $\ell_{V}$ only influence the description of the leptonic decays of neutral vector mesons.

The explicit form of this Lagrangian is given in the Appendix. In principle, from this Lagrangian and the non-anomalous $\mathcal{L}_{V}$ Lagrangian piece given in Eq. (14), one is able to construct decay amplitudes for the processes $V \rightarrow P \gamma, P \rightarrow V \gamma, V \rightarrow e^{+} e^{-}$and $P \rightarrow \gamma \gamma$. 


\section{RADIATIVE AND LEPTONIC DECAYS OF LIGHT MESONS}

Using the Lagrangian of Eq. (18) and the piece in Eq. (14), we can get the coupling constants for the decays $V \rightarrow P \gamma$ and $P \rightarrow V \gamma$, given by Eqs. (A3) and (A4). They are related to the partial decay widths through

$$
\left\{\begin{array}{l}
\Gamma(V \rightarrow P \gamma)=\frac{1}{96 \pi}\left[\frac{m_{V}^{2}-m_{P}^{2}}{m_{V}}\right]^{3}\left|G_{V P \gamma}\right|^{2} \\
\Gamma(P \rightarrow V \gamma)=\frac{1}{32 \pi}\left[\frac{m_{P}^{2}-m_{V}^{2}}{m_{P}}\right]^{3}\left|G_{V P \gamma}\right|^{2}
\end{array}\right.
$$

Eq. (A5) gives the connection between the main coupling parameter $G$ in Ref. [5] and more usual quantities like the pion decay constant $f_{\pi}$ and the universal vector meson coupling $g$, introduced by the covariant derivative in Eq. (5).

Some of these couplings are totally unaffected by any breaking process (such as all $G_{V \pi \gamma}$ ), while some are affected only by broken nonet symmetry (like $G_{\rho^{0} \eta \gamma}$ and $G_{\rho^{0} \eta^{\prime} \gamma}$ ). In couplings involving both isoscalar vector and pseudoscalar mesons, the two $\mathrm{SU}(3)$ breaking mechanisms accounted for above by $\ell_{A}(\mathrm{BKY})$ and $\ell_{W}(\mathrm{BGP})$, come mixed together in a way which could prevent one from separately testing the relevance of the two kinds of breakings $\left(Z=\ell_{W} / \ell_{A}\right)$. However, in the radiative decays of the $K^{*}$ mesons, one is able to extract information about these two parameters as they act differently for the two different charged modes. This is fortunate, since, otherwise, there would be no way to disentangle what comes from $\ell_{A}$ (and thus from $f_{K}$ ) from what comes from $\ell_{W}$. In order to compare with recent modellings, we see for instance that the relation $G_{\rho^{0} \eta \gamma} / G_{\rho^{0} \eta^{\prime} \gamma}=\tan \delta_{P}$ 25] is modified by nonet symmetry breaking.

One can check that the coupling constants deduced from the Lagrangian in Eq. (16), supplemented by the replacement $\eta_{0} \longrightarrow x \eta_{0}$, are in perfect agreement with the exact $\mathrm{SU}(3)$ model of O'Donnell [4. Indeed, setting $\ell_{W}=\ell_{A}=1$ in Eqs. (A3) and (A4), allows one to recover the expected expressions for the coupling constants [4,5], when assuming nonet symmetry in the vector sector only. From this point of view, the breaking scheme provided by the BKY and BGP mechanisms, happens to be non-trivial, as can be seen from Eqs. (A3) and (A4). For instance, it should be noted that decays involving $\eta$ and $\eta^{\prime}$ mesons are not simply rescaled in the breaking procedure, but that the breaking procedure treats differently strange and non-strange contributions to the coupling constants.

Following standard rules, the same Lagrangian information allows us to reconstruct definite expressions (see Eq. (A6)) for the two-photon couplings of the pseudoscalar mesons; these are related to partial widths by

$$
\Gamma(X \rightarrow \gamma \gamma)=\frac{M_{X}^{3}}{64 \pi}\left|G_{X \gamma \gamma}\right|^{2} \quad, \quad X=\pi^{0}, \eta, \eta^{\prime}
$$

Let us first remark that the expression for $G_{\eta \gamma \gamma}$ compares well with the corresponding expression of Ref. [52] deduced from the Nambu-Jona-Lasinio model, showing that breaking parameters in this reference, originally expressed as functions of effective quark masses, also 
get an expression? in terms of $f_{\pi} / f_{K}$. More interesting is that we recover the traditional form for these amplitudes, (i.e. the one mixing angle expressions [15 17,20,21]). Using these standard expressions, one indeed gets through identification

$$
\frac{f_{\pi}}{f_{8}}=\frac{5-2 Z}{3}, \quad \frac{f_{\pi}}{f_{1}}=\frac{5+Z}{6} x
$$

where $Z=\ell_{W} / \ell_{A}$. This shows that, in the limit of SU(3) symmetry, we have $f_{8}=f_{\pi}$ and $f_{1}=f_{\pi} / x$, and, that $f_{1}=f_{8}=f_{\pi}$ supposes that there is no symmetry breaking at all. It is interesting to note that the FKTUY Lagrangian [10], broken as we propose, expresses all decay constants in terms of $f_{\pi}, f_{K}, x$ and the BGP breaking parameter $\ell_{W}$ (if these last two quantities are found to depart significantly from unity). It should anyway be noted that $f_{1}$ and $f_{8}$ get expressions in terms of parameters which can be calibrated outside the set of $P \rightarrow \gamma \gamma$ decays. Eq. (21) shows that the BGP breaking parameter is connected to the relation between $f_{8}, f_{K}$ and $f_{\pi}$.

Concerning leptonic decay widths, if one relies on the model described above and in the Appendix, a combination of the $f_{V \gamma}$ is unaffected by $\mathrm{SU}(3)$ breaking and can be written $f_{\omega \gamma} \cos \delta_{V}+f_{\phi \gamma} \sin \delta_{V}=f_{\rho \gamma} / 3$, or alternatively

$$
f_{\omega \gamma} G_{\omega \pi^{0} \gamma}+f_{\phi \gamma} G_{\phi \pi^{0} \gamma}=f_{\rho \gamma} G_{\rho^{0} \pi^{0} \gamma}
$$

in terms of measured quantities only. Correspondingly, the $X_{V}$ breaking is fully concentrated in another independent combination $f_{\phi \gamma} \cos \delta_{V}-f_{\omega \gamma} \sin \delta_{V}=\left(\sqrt{2} f_{\rho \gamma} / 3\right) \ell_{V}$. This relation can be rewritten in terms of measured quantities only

$$
\ell_{V}=\frac{1}{\sqrt{2}} \frac{f_{\phi \gamma} G_{\omega \pi^{0} \gamma}-f_{\omega \gamma} G_{\phi \pi^{0} \gamma}}{f_{\phi \gamma} G_{\phi \pi^{0} \gamma}+f_{\omega \gamma} G_{\omega \pi^{0} \gamma}}
$$

Therefore, one has, as an alternative to mass relations [11, a coupling relation to define $\ell_{V}$. Additionally, the HLS parameter $a$ fulfills

$$
a=\frac{e}{8 \pi^{2} f_{\pi}^{3}} \frac{f_{\rho \gamma}}{G_{\rho^{0} \pi \gamma}}
$$

and can be extracted in this way from data; however, it seems more accurate to perform a global fit of all coupling constants involving leptons or photons.

Before closing this section, one practical remark could be of interest. When computing, for instance, the decay amplitude $\pi^{0} \rightarrow \gamma \gamma$, one is tempted to consider only the leading order terms which can be written symbolically

$$
A\left(\pi^{0} \longrightarrow\left[\rho^{0} \rightarrow \gamma\right][\omega \rightarrow \gamma]\right)+A\left(\pi^{0} \longrightarrow\left[\rho^{0} \rightarrow \gamma\right][\phi \rightarrow \gamma]\right)
$$

and possibly add up the next order terms

\footnotetext{
${ }^{9}$ This might indicate that some relation exists connecting effective masses of quarks and meson decay constants.
} 


$$
A\left(\pi^{0} \longrightarrow\left[\rho^{0} \rightarrow \gamma\right][\omega \rightarrow \phi \rightarrow \gamma]\right)+A\left(\pi^{0} \longrightarrow\left[\rho^{0} \rightarrow \gamma\right][\phi \rightarrow \omega \rightarrow \gamma]\right),
$$

by taking into account the $\omega \leftrightarrow \phi$ direct transition term given in Eq. (14). In doing this way, we reach the surprising conclusion that the decay width $\pi^{0} \rightarrow \gamma \gamma$ is affected by breaking the $\mathrm{SU}(3)$ symmetry. Actually, this is an artefact which can be circumvented by summing up (formally) the full series of such $\omega \leftrightarrow \phi$ transitions and, in this case, we indeed get the third Eq. (A6), exactly as if one uses the $\omega^{I}$ and $\phi^{I}$ combinations for virtual lines (i.e. for lines going from a decay vertex to the transition to photons). This remark applies in computing any decay amplitude involving $\omega$ and $\phi$ legs hidden beneath each $\gamma$ external leg.

\section{FITTING DECAYS MODES WITH THE BROKEN MODEL}

In this section, we focus on the model for coupling constants given by Eqs. (A3) to (A7), i.e. we make the assumption that there is no glue hidden inside the $\eta$ or $\eta^{\prime}$ mesons. The quantities submitted to fit are the (measured) coupling constants which can be deduced from the partial widths in the Review of Particle Properties [30], using formulae recalled above.

\section{A. Comments on Radiative $K^{*}$ Decay Measurements}

As commented above (see Section [1), there are potential problems with both $K^{*}$ decay modes. Therefore, we have followed the strategy of performing fits of all radiative decay modes except for these two. Then, the fit values of the free parameters allow us to predict a value for the partial widths $K^{* 0} \rightarrow K^{0} \gamma$ and $K^{*+} \rightarrow K^{+} \gamma$, making it possible to compare the $\chi^{2}$ distance of each these predicted values to the corresponding measured values [30]. In all fits, we have found that the prediction for $K^{* 0} \rightarrow K^{0} \gamma$ is in fairly good agreement with the corresponding measurement, while the expected value for $K^{*+} \rightarrow K^{+} \gamma$ is always at about $5 \sigma$ from the accepted value [30], casting some doubt on the reliability of this measurement. Therefore, in the fits referred to hereafter, the process $K^{*+} \rightarrow K^{+} \gamma$ has been removed. We shall nevertheless reexamine this question in detail in Section X1.

\section{B. The HLS Parameter $a$ and the Breaking Parameter $\ell_{V}$ from Leptonic Decays}

Among the quantities we fit, most depend on only $G, x, \ell_{A}, \ell_{W}$ and the mixing angles $\theta_{V}$ and $\theta_{P}$ : these are the radiative decays $V \rightarrow P \gamma, P \rightarrow V \gamma$, and the decays $P \rightarrow \gamma \gamma$, and they represent the most important part of the broken symmetry model we have built.

On another hand, the leptonic decay modes of the vector mesons are interesting because they open a new window for estimating the HLS parameter $a$ and the breaking parameter $\ell_{V}=\left(1+c_{V}\right)^{2}$. We could, as well, have used the combination in Eq. (22) which does depend neither on $\ell_{V}$ nor $a$. We have, however, preferred keeping the three modes $\rho^{0} / \omega / \phi \rightarrow e^{+} e^{-}$, in order to get new determinations of $\ell_{V}$ and $a$.

Without going into too much detail, it happens (as could been foreseen from the very existence of Eqs. (23) and (24)), that values for these parameters do not depend in any way on assumptions about the other sectors, nor even on the global fit quality level. 
In this way, the values provided by both radiative and leptonic decays for these parameters are

$$
a=2.50 \pm 0.03 \quad, \quad \ell_{V}=1.38 \pm 0.03 \quad\left(c_{V}=0.173 \pm 0.013\right)
$$

This value for $a$ (quite inconsistent with the standard VMD expectation $a=2$ ) should be compared with the value reported from fitting $e^{+} e^{-} \rightarrow \pi^{+} \pi^{-}$data of Ref. [53] in Ref. [46] $(a=2.37 \pm 0.02)$, and with the fit of the preliminary data collected by the new CMD2 detector [47] $a=2.35 \pm 0.02$. The final analysis of this last data set is now available [54] and provides $a=2.38 \pm 0.02$.

Even if comparable, one observes a significant difference (about $3 \sigma$ ) and its origin is unclear. However, one should notice that the value of $a$ measured from $e^{+} e^{-} \rightarrow \pi^{+} \pi^{-}$is determined by the magnitude of a non-resonant contribution to the scattering amplitude, while the value in Eqs. (25) is determined by meson decays. This could be a signal of an additional $\mathrm{SU}(2)$ breaking or of some systematic errors hard to identify presently.

The value for $c_{V}$ differs by a factor of two from what would be expected if we use the mass formulae [11]. However, it seems that the data on radiative and leptonic decays favor the relation suggested in Ref. [12], $c_{A}=a c_{V}$. It should be useful to check whether or not this is a numerical accident, for instance, by a detailed study of the annihilation processes $e^{+} e^{-} \rightarrow K \bar{K}$.

\section{The SU(3) Breaking Parameters $\ell_{A}$ and $\ell_{W}$}

Two of the key parameters in the broken $\mathrm{SU}(3)$ model we use in describing the radiative decays of light flavor mesons, are the BKY parameter [11,12] $\ell_{A}$ expected to be equal to $\left(f_{K} / f_{\pi}\right)^{2}$ and the BGP parameter $\ell_{W}$.

As commented in the Appendix, the dependence upon $\ell_{A}$ and $\ell_{W}$ is always through their ratio (which is already referred to as $Z$ ) except for the $K^{* 0}$ decay mode expect large correlations between $\ell_{A}$ and $\ell_{W}$. From a first fit with these parameters both free, we obtain

$$
\ell_{W}-1=c_{W}=\left(-0.17_{-0.35}^{+0.59}\right) \times 10^{-3}
$$

with a nice fit probability $\left(\chi^{2} / \mathrm{dof}=10.74 / 8\right)$. Therefore we have performed the fit setting $\ell_{W}=1$ (or $c_{W}=0$, i.e. no BGP breaking), and found unchanged fit quality $\left(\chi^{2} / \mathrm{dof}=\right.$ 10.74/9). Thus we may conclude that there is no need for the BGP breaking mechanism at a level visible in the existing data $\square$. In this last fit, we also get

$$
\ell_{A}=1.480_{-0.047}^{+0.049}
$$

\footnotetext{
${ }^{10}$ We remind the reader, that $K^{* \pm}$ decay mode is anyway inconsistent with the other data and has been removed from the fit set.

${ }^{11}$ See however Section XI.
} 
which is almost exactly the value expected from the known ratio $f_{K} / f_{\pi}$ (see Eq. (12)). This gives, of course, a strong support to the breaking mechanism proposed by Bando, Kugo and Yamawaki [11, 12]. Indeed, the relation between $\ell_{A}$ and $f_{K} / f_{\pi}$, which is mandatory within the BKY breaking scheme in order to fulfill $F_{K^{+}}(0)=1$, even after $\mathrm{SU}(3)$ breaking, is found here to hold numerically to quite a nice precision. It should be noted that it comes together with either nonet symmetry breaking $(x \neq 1)$ or glue inside the system $\eta / \eta^{\prime}$, as will be commented on below.

At this point, a comment is of relevance about the results reported in Ref. [6]. Our result in Eq. (26), means that taking into account all radiative decay modes of light mesons, forces one to remove the data for $K^{* \pm}$ decay mode as it is inconsistent with all the rest; in this case the BGP breaking practically cancels out. By taking a part of the decay modes only, the relative inconsistency of the data for both $K^{*}$ 's is not obvious and explains the result of Ref. [6].

One could consider the result in Eq. (27) as providing an interesting estimate of $f_{K} / f_{\pi}$, independent of measurements of $K$ and $\pi$ decays

$$
\frac{f_{K}}{f_{\pi}}=1.217_{-0.019}^{+0.021}
$$

The results metioned above suggest that one can reasonably fix $\ell_{A}=1.50$ (at its physical value), and remove the BGP breaking $\left(\ell_{W}=1\right)$ which happens to be useless, as soon as the BKY breaking is correctly set up. Then, the single free breaking parameter which influences the coupling constants in radiative decays, beside mixing angles, is the nonet symmetry breaking parameter $x$.

\section{The Nonet Symmetry Breaking Parameter $x$}

Therefore, the preliminary fits sketched above allow us to conclude that the only actual free symmetry breaking parameter is $x$, once we do not consider a coupling of the $\eta / \eta^{\prime}$ doublet to glue. Stated otherwise, except for the two mixing angles, we only have two free parameters to fit the data set, as in the unbroken case [5]. One, named $G$, is connected with the vector meson universal coupling $g$, the other is the nonet symmetry breaking parameter $x$. The former is clearly fundamental $(G)$ while it is uncertain whether or not the latter should be considered fundamental.

We give in the first data column of Table $\mathbb{E}$, the fit results assuming nonet symmetry $(x=1)$, and leaving free all other model parameters. The best $\chi^{2} /$ dof we reach is $30 / 9$, showing that the BKY and BGP breaking mechanisms alone are unable to mimic a violation of nonet symmetry in the pseudoscalar sector. The mixing angle is at a value frequently obtained in this case [5, 8, 13, 55, 56]; however, the fit probability is low enough $\left(5 \times 10^{-4}\right)$, that the assumption of full nonet symmetry can be considered sharply disfavored.

In the second data column in Table I, we display the fit results, assuming fixed $\mathrm{SU}(3)$ breaking conditions $\left(\ell_{A}=\left(f_{K} / f_{\pi}\right)^{2}\right.$ and $\left.\ell_{W}=1\right)$; in this case, we get instead a very nice fit probability (44\%). This fit provides $x=0.92 \pm 0.02$, and indicates a significant departure from nonet symmetry $(\simeq 4 \sigma)$. More appealing is the mixing angle of pseudoscalar mesons coming out from fit : $\theta_{P}=-11.59^{\circ} \pm 0.76^{\circ}$, in perfect agreement with the linear mass formula, which predicts $-10.1^{\circ}$. 
One should also notice that the vector mixing angle is found to be $3.4^{\circ}$ smaller than its ideal value, in agreement with the prediction of Ref. [57] and previous fit results [5], keeping in mind, however, that this sign for departure relative to ideal mixing depends on the definition of the ideal $\phi$, and that we use $\phi^{I}=-s \bar{s}$.

The value for $G=0.704 \pm 0.002 \mathrm{GeV}^{-1}$ is remarkably stable, independently of fit con-

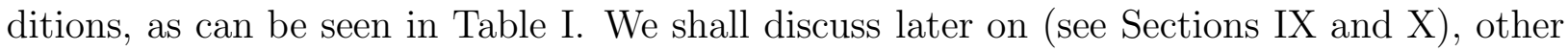
consequences of the value found for $G$, which is also in perfect agreement with the result obtained [5] assuming no $\mathrm{SU}(3)$ breaking symmetry. This is not surprising, as $G$ is practically determined by all $\rho$ decay modes and the $\phi$ and $\omega$ decays to $\pi^{0} \gamma$, that is from a large number of processes not affected by SU(3) breaking (see Eqs. (A3) and (A4)).

\section{E. The One Angle $\eta / \eta^{\prime}$ Mixing Scheme from VMD}

As discussed above, and in the Appendix, the model we propose, which relies on the VMD approach of Refs. [9, 10], with fixed $\mathrm{SU}(3)$ breaking à la BKY [11,12], leads to (one angle) formulae for the $\eta / \eta^{\prime} \rightarrow \gamma \gamma$ decay amplitudes, which can be identified with the corresponding Current Algebra standard expressions. This justifies the identification shown in Eq. (21) for the singlet and octet coupling constants. One should note that nonet symmetry breaking does not conceptually modify the formulae substantially.

In this case, we obtain together with $\theta_{P}=-11.59^{\circ} \pm 0.76^{\circ}$

$$
\frac{f_{8}}{f_{\pi}}=0.82 \pm 0.02 \quad, \quad \frac{f_{1}}{f_{\pi}}=1.15 \pm 0.02
$$

using Eq. (27), and the fit result for $x$.

One should note that the value obtained for $f_{8} / f_{\pi}$ is not in agreement with the ChPT numerical expectation [50,58]. This is a quite mechanical consequence of the low value we get from our fit for the mixing angle, as can be read off Fig. 1 in Ref. [13]. However, this does not prevent VMD from providing quite a satisfactory description of all observables associated with light meson decays, including a nice fit value of $\ell_{A} \simeq f_{K} / f_{\pi}$, as seen above and as will be illustrated below.

One should also note that relatively low values of $\theta_{P}$ have been advocated (or found) in analyzing similar data, for instance in Refs. [13,24,56]. It can thus be remarked that in the one mixing angle approach, it is only the addition of $J / \psi$ decays which pushes $\left|\theta_{P}\right|$ to larger values.

\section{F. Radiative Decays versus $\gamma \gamma$ Decays}

The results reported in the previous subsections mix $P V \gamma$ and $P \gamma \gamma$ couplings. In connection with recent works [22,23,26], one could ask whether the good description obtained using only one mixing angle, $\theta_{P}$, is not merely an artefact produced by merging these two kinds of couplings in a single fit procedure. In order to test this point, we have performed fits of the $P V \gamma$ processes in isolation. 
In a first fit, we removed the $K^{* 0}$ mode, in order to have a reference without any influence of $K^{*}$ decay modes. Fixing, as usual[ ${ }^{\mathrm{Z}} \ell_{A}=1.5$ and $\ell_{W}=1$, we get a nice fit $\left(\chi^{2} / \mathrm{dof}=8.6 / 7\right)$, finding that the observed value for $K^{* 0} \rightarrow K^{0} \gamma$ was only $0.57 \sigma$ from the predicted value, while the data for $K^{* \pm} \rightarrow K^{ \pm} \gamma$ was $4.84 \sigma$ from expectation. Therefore, it was indeed meaningful to include the $K^{* 0}$ data in the fit, which is practically unchanged $\left(\chi^{2} / \mathrm{dof}=\right.$ $8.9 / 7)$.

In this case, we also found that the measured value for $\eta \rightarrow \gamma \gamma$ was $2.18 \sigma$ from the prediction of Eq. (A6), while the data for $\eta^{\prime} \rightarrow \gamma \gamma$ was $0.50 \sigma$ from the corresponding prediction. In this last fit, the parameter values we get are practically indistinguishable from the information in the second data column of Table \; the value for $\theta_{P}$ was, by far, the most sensitive ${ }^{\mathrm{T3}}$ and moved to $-10.41^{\circ} \pm 1.21^{\circ}$, that is only by $\sim 0.5 \sigma$.

From this we can conclude that the two-photon decays for $\eta$ and $\eta^{\prime}$ are fully predicted from $V P \gamma$ processes, which can be considered as a considerable success of the FKTUY Lagrangian [10], supplemented by the BKY breaking mechanism [11, 12] set at a fixed value, and of broken nonet symmetry.

One might ask oneself whether our result about $f_{8}$ (which mechanically follows from simply the BKY breaking scheme) is really in contradiction with the ChPT expectation $f_{8} \simeq 1.25 f_{\pi}$. The answer is presently unclear because the definition we use for the decay constants proceeds from the Wess-Zumino-Witten Lagrangian [20,21], as reexpressed in Refs. 15 17, and not on the coupling of pseudoscalar mesons to an axial field. This question will be examined in a forthcoming paper.

As a conclusion, within the context of light meson decays, we find no failure of the VMD approach sketched above ${ }^{\mathbb{t}}$ and no need for a second angle [22,23,26] arises naturally from the data examined so far. However, one cannot exclude that nonet symmetry breaking is somehow equivalent to this second angle. This does not seem easy to prove from standard algebra.

\section{NONET SYMMETRY BREAKING VERSUS GLUE}

Up to now, we have clearly illustrated that the BKY breaking was a fundamental tool in order to describe all data concerning radiative and two-photon decays of light mesons. Correspondingly, we have shown that, at the level of refinement allowed by the data, there was no need for the additional BGP breaking.

Another central result of our fitting model concerns the unavoidable need of about $10 \%$ breaking of nonet symmetry in the pseudoscalar sector $(x \simeq 0.9)$. Even if small, this should be considered significant, as it is more than a $4 \sigma$ effect. This could well be a property that has to be accounted for at a fundamental level. However, nonet symmetry breaking

\footnotetext{
${ }^{12}$ In this case, we are actually only sensitive to the ratio of breaking parameters $Z=\ell_{W} / \ell_{A}$.

${ }^{13}$ This simply reflects that Eqs. (A6) only depend on the mixing angle.

${ }^{14}$ The problem with the $K^{* \pm}$ radiative decay can be solved ad minima as will be shown in Section $\mathrm{XI}$.
} 
could well be an effective way to account (maybe partly at least) for another physical effect, ignored in the model presented above.

We have already discussed the possible interplay of an additional singlet component which could be present inside the $\eta / \eta^{\prime}$ system. This could be a gluonium component, a coupling to $c \bar{c}$ or a mixture of both. It is not the purpose of the present paper to try identifying this third component and the corresponding partner of the $\eta$ and $\eta^{\prime}$ mesons. Our concern is rather to see whether a coupling to such an additional singlet could play some role in the problem we examine.

The coupling to this additional state (hereafter named glue, somehow abusively) has been presented in Section III and is summarized by Eq. (2). The corresponding coupling constants, as far as they are affected, are explicitly given by Eqs. (A9) to (A11).

A preliminary study of these relations, which include both $\mathrm{SU}(3)$ breaking effects, nonet symmetry breaking and glue has been performed. The conclusions are twofold:

- The previous conclusions concerning the BKY and BGP breaking mechanisms are unchanged. More precisely, the BKY breaking is found determined by the value of $f_{K} / f_{\pi}$, while a possible BGP breaking is found too small to be observed. Numerically, all conclusions of the previous section remain fully valid.

- Nonet symmetry breaking and glue are intimately connected and reveal a correlation close to the $100 \%$ level.

The second point does not mean that nonet symmetry breaking is physically equivalent to assuming coupling to glue, but numerically it is indeed so. It also tells that, if we know what is the precise amount of glue, one can deduce the level of nonet symmetry breaking (or conversely).

Table 【 Iisplays the main results of fitting the data with a fixed level of symmetry breaking. We remind that $\beta$ governs the level of glue inside the $\eta$ meson, while $\gamma$ governs the level of glue inside the $\eta^{\prime}$ meson. It is clear from this table, that $x$ cannot be too small ; for $x \simeq 0.8$ and smaller values, glue is unable to account for the two-photon decays and $\eta^{\prime}$. Values larger than $x \simeq 0.9$ look all statistically acceptable, except that $\theta_{P}$ becomes less and less negative, and even changes its sign! Moreover, the glue content needed in $\eta$ increases slowly from zero (at $x=0.9$ ) to an arbitrary value.

This clearly illustrates that above some level for $x$ (about 0.85 ), one cannot distinguish the effects of glue from those of a genuine nonet symmetry breaking effect.

Two interesting features are however visible in Table [I] :

- At $x \simeq 0.9$ both $\beta$ and $\gamma$ are quite consistent with zero. This indicates that $x \simeq 0.9$ implies a decoupling of the glue from $\eta$ and $\eta^{\prime}$ mesons. This is the case developed in the preceding Section.

\footnotetext{
${ }^{15}$ At $x=0.7$ the predicted amplitudes for $\eta / \eta^{\prime} \rightarrow \gamma \gamma$ are both at $7 \sigma$ from the measured values ; at $x=0.8$, the disagreement is still about $4 \sigma$ each.
} 
- In the case of full nonet symmetry $x \simeq 1.0$, it is interesting to remark that $\beta$ is still consistent with zero, pointing to the fact that nonet symmetry implies that $\eta$ couples only to the standard quarkonium states named $v_{8}$ and $v_{1}$ in Section III. In this case however, the glue content of the $\eta^{\prime}$ meson becomes significant.

In view of these results, it looks justified to perform a fit, fixing (as before) the $\mathrm{SU}(3$ ) breaking effects to $\ell_{A}=1.5, \ell_{W}=1, x=1$ and also $\beta=0$ (in order to lessen at most correlation effects). In this case we have exactly the same number of parameters as in the previous set of fits. The corresponding fit results are also displayed in Table $\mathbb{1}$ and show a nice quality $\left(\chi^{2} / d o f=10.5 / 10\right)$, equivalent to the no-glue case.

The glue content this implies for the $\eta^{\prime}$ meson can be presented in several ways. Writing $\eta^{\prime}=X v_{8}+Y v_{1}+Z v_{1}^{\prime}$ (with $X^{2}+Y^{2}+Z^{2}=1$ ), we have $\sqrt{X^{2}+Y^{2}}=0.89$ and $Z=0.46$. One can express the glue fraction as $Z^{2}=\cos ^{2} \gamma \simeq 0.20$ (at $x=1$ ).

As major conclusions of this section, one can first assert that a possible glue content inside the $\eta$ is not requested by the data. A significant glue content inside the $\eta^{\prime}$ is however subject to the actual level of nonet symmetry breaking.

We do not discuss any more values and meaning of $f_{1}$ and $f_{8}$. Eq. (A11), indeed shows that the meaning of these has to be revisited. Moreover, the specific two-angle formulation of the $\eta / \eta^{\prime} \rightarrow \gamma \gamma$ decays introduced by the glue coupling $\left(\theta_{P}\right.$ and $\left.\gamma\right)$, appears quite different from the one introduced in Ref. [22, 23].

\section{PRIMAKOFF VERSUS $e^{+} e^{-}$INFORMATION FOR $\eta$ DECAY}

All fit results presented so far, assumed the use of the partial width $\eta \rightarrow \gamma \gamma$ as obtained in $e^{+} e^{-}$experiments. As mentioned above, this looks a priori better grounded, as the number of $e^{+} e^{-}$experiments is large ; this kind of experiments looks also more straightforward to interpret.

However, in case where the (single) measurement relying on the Primakoff effect might have to be considered, it is not completely useless to examine rapidly its consequences.

We have fit under the two assumptions of nonet symmetry breaking (and no glue) and coupling to glue (with no nonet symmetry breaking). All other parameters have been set at their usual values. The results are given in the last two data columns of Table [1.

The first remark is that the fits in both cases work as well as when using the $e^{+} e^{-}$ information for $\eta$. A few undesirable features are :

- The mixing angle $\theta_{P}$ is significantly smaller (in absolute magnitude) with the Primakoff measurement than with the $e^{+} e^{-}$data.

- Replacing nonet symmetry breaking by glue, leads to glue content in $\eta$ as large as in the $\eta^{\prime}$, if we estimate its fraction by the angles $\beta$ and $\gamma$ ).

The second item above might dismiss physically the reliability of the Primakoff Effect measurement of $\eta \rightarrow \gamma \gamma$. 


\section{ESTIMATES FOR BRANCHING FRACTIONS FROM FITS}

The fits we have performed provide under various symmetry breaking conditions, the

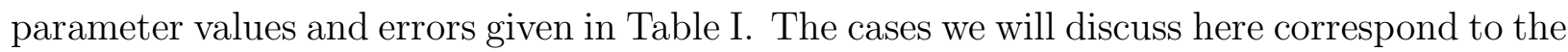
second and third data columns in Table I, which both give a very good fit quality. These are the cases with i/ nonet symmetry breaking supplemented by a fixed SU(3) breaking (BKY) and ii/ a fixed $\mathrm{SU}(3)$ breaking $(\mathrm{BKY})$ with glue inside the $\eta^{\prime}$ replacing nonet symmetry breaking. We now compare the branching fractions predicted by these two solutions to the accepted branching fractions as given in the Review of Particle Properties [30]. They are computed using the formulae for coupling constants given in the Appendix and the relations defining the partial widths. The coupling constants just referred to are computed from the basic parameters $\left(G, x, \theta_{P}, \theta_{V}, \beta, \gamma\right)$, by identifying these with gaussian distributions having as mean values the central values in the fit and as standard deviations, the corresponding $(1 \sigma)$ error.

In Table [II, we first list the information for radiative decays only. The first remark which comes to mind by comparing the two models is that their predictions are close together. The relative disagreement with $\eta^{\prime} \rightarrow \rho^{0} \gamma$ is actually an artefact. Indeed, what has been fit is not the branching fraction given in Ref. [30], but the corresponding coupling constant which has been extracted by the Crystal Barrel Collaboration in [60]. The reason for this is that the (published) branching fraction for $\eta^{\prime} \rightarrow \rho^{0} \gamma$ is influenced by the box anomaly [5, 15, 17, 20, 21 for the vertex $\eta^{\prime} \pi^{+} \pi^{-} \gamma$ which is not accounted for in the VMD model of [10]; actually this process contributes to the $\chi^{2}$ for only $\simeq 0.5$.

On the other hand, the PDG information reported for $\eta \rightarrow \gamma \gamma$ branching fraction is the official one [30], somehow influenced by the Primakoff measurement.

The single clear disagreement of model predictions with data concerns the branching fraction for $K^{* \pm} \rightarrow K^{ \pm} \gamma$, that we find about half of the reported value in the Review of Particle Properties [30]. We postpone to Section XI the reexamination of this question.

Otherwise, the largest disagreement is never greater than about $1.5 \sigma$. At such a (nonsignificant) level, it is hard to distinguish whether differences between predictions and data are due to $\mathrm{SU}(2)$ breaking effects missing in the models, to systematic errors in the data or to the (unavoidable) influence of the resonance models used to extract branching fractions from data. For instance, changing the model for the $\rho$ lineshape in the cross section for $e^{+} e^{-} \rightarrow \pi^{0} \gamma$ allows to reduce the branching ratio for $\rho^{0} \rightarrow \pi^{0} \gamma$ from $(6.8 \pm 1.7) 10^{-4}$ to $(6.1 \pm 1.5) 10^{-4}$ which compares better to the corresponding prediction $\left(5.210^{-4}\right)$.

The new measurement for $\phi \rightarrow \eta^{\prime} \gamma$ is also well accepted by the fit. However, the prediction tends to indicate that the central value found by SND Collaboration [35] is favored compared to that of the CMD2 Collaboration [34].

Additionally, Table IV shows that leptonic decays of vector mesons are very well described.

From all this, we can conclude that the model of symmetry breaking, we have presented provides a consistent description of the data. At their present level of accuracy, these do not seem to require additional symmetry breaking effects.

An especially satisfactory conclusion is that $\mathrm{SU}(3)$ breaking effects are not left free in the fits and are practically determined by the ratio $f_{K} / f_{\pi}$. Some nonet symmetry breaking in the pseudoscalar sector is, however, requested by the data. This is fully or partly degenerated 
with a possible admixture of glue, shown to (possibly) affect only the $\eta^{\prime}$ meson. If this has to be seriously considered, the question is to identify the third partner to the $\left(\eta, \eta^{\prime}\right)$ doublet which has been named $\eta^{\prime \prime}$. For this purpose, a precise study of the decay properties of the $\eta(1440)$ meson could improve the hint. One has also to mention that this glue component could be a $c \bar{c}$ admixture.

\section{HADRONIC DECAYS OF VECTOR MESONS}

From the above fits of radiative decays, we get stable estimates for the HLS parameters $a(2.5 \pm 0.03)$ and $g(5.65 \pm 0.02)$ and also for the breaking parameter $\ell_{V}(1.38 \pm 0.03)$. The deviation from ideal mixing is $\delta_{V}=-3.33 \pm 0.16$ degrees. In this Section, we use the fir results obtained assuming nonet symmetry breaking and $\mathrm{SU}(3)$ breaking with $\ell_{A}=1.5$ (second data column in Table (1)).

If one relies on the $\mathrm{SU}(3)$ broken HLS non-anomalous Lagrangian [12], using these parameter values, one can extract the coupling constants $g_{\rho \pi \pi}$ and also, for instance, the coupling constants $g_{\phi K^{+} K^{-}}$and $g_{\phi K^{0} \bar{K}^{0}}$ and compare to the corresponding data.

A value for $g$ can be extracted from fit to the $e^{+} e^{-} \rightarrow \pi^{+} \pi^{-}$data [46], using $G_{\rho \pi \pi}=a g / 2$ and one gets $g=5.18 \pm 0.02$. This value compares poorly to the value we just get using radiative decays of light mesons $(5.65 \pm 0.02)$. This means that, from radiative decays, one might expect a broader width for the $\rho$ meson. However, the coupling constants for the $\phi$ carry the same information as $\rho^{0}$. Indeed, it is easy to get using Ref. [12]

$$
G_{\phi K \bar{K}}=-\frac{a g}{4 \ell_{A}}\left[\sin \delta_{V}-\sqrt{2} \ell_{V} \cos \delta_{V}\right]
$$

for both possible final states, $K^{+} K^{-}$and $K^{0} \bar{K}^{0}$. From standard formulae this coupling constant provides the branching fractions shown in Table IV] which compare quite well to the data.

Therefore, in contrast with the hadronic width of the $\rho$, the hadronic width of $\phi$ is in nice agreement with the value found for $g$ when fitting radiative decays $(5.65 \pm 0.02)$, with symmetry breaking parameters only influenced by leptonic decays of vector mesons.

One can attempt the same comparison with $K^{*}$ hadronic decays to $K \pi$ (as studied in Refs. [38] and [59]). In this case, the coupling constants can be read off the Lagrangian given in Ref. [12]. Expliciting the breaking parameter dependence, they are

$$
\left\{\begin{array}{l}
g_{K^{* 0} K^{0} \pi^{0}}=\frac{g_{K^{* 0} K^{+} \pi^{-}}}{\sqrt{2}}=\frac{a g}{4} \sqrt{\frac{\ell_{V}}{\ell_{A}}} \\
g_{K^{* \pm} K^{ \pm} \pi^{0}}=\frac{g_{K^{* \pm} K^{0} \pi^{ \pm}}}{\sqrt{2}}=\frac{a g}{4} \sqrt{\frac{\ell_{V}}{\ell_{A}}}
\end{array}\right.
$$

Using the experimental data [30], $\ell_{A}=1.5$, and the fit values for the parameters $a$ and $\ell_{V}$, one can extract $g=5.86 \pm 0.10$ (neutral $K^{*}$ ) and $g=5.98 \pm 0.11$ (charged $K^{*}$ ) which compare relatively well to our fit value $(5.65 \pm 0.02)$, even if it is not as good as for the $\phi$ meson hadronic decays.

This quite unexpected situation seems likely to be connected to the issue of defining masses and widths for broad resonances like the $\rho$ and $K^{*}$ mesons. To be more precise, this 
addresses the question of the connection between these physical parameters extracted from data, obtained using Breit-Wigner resonance lineshapes, and the corresponding quantities occurring in a Lagrangian for broad resonances [61. This question will be studied in a forthcoming paper.

\section{THE $K^{* \pm}$ RADIATIVE DECAY PROBLEM}

The question of whether the radiative decay $K^{* \pm} \rightarrow K^{ \pm} \gamma$ is definitely beyond the scope examined so far should be answered.

The first point which comes to mind is whether the disagreement reported above (a factor of two between prediction and measurement) could be explained by breaking the SU(2) flavor symmetry. The answer is seemingly no ; indeed, taking into account the quark content of the $K^{*}$ 's, one could rather guess that a significant unaccounted for $\mathrm{SU}(2)$ breaking would affect the quality of predictions for $K^{* 0}$ rather than for $K^{* \pm}$. However, the absolute partial width of the $K^{* 0}$ is well predicted by our modellings (flavor $\mathrm{SU}(3)$ and nonet symmetry breakings and/or glue).

This possibility seeming unlikely, the question becomes : can the modelling developed in the Appendix be modified in order to account for this mode within an extended SU(3) breaking framework? The reply is positive and is the following.

Within the spirit of the BKY mechanism, the (unbroken) FKTUY Lagrangian given in Eq. (16) can be broken straightforwardly in three different ways. The first mean is the pseudoscalar field renormalization (see Eq. (17)), which leads to introduce the matrix $X_{A}$ and thus the breaking parameter $\ell_{A}$ expected [11] and found (see Eq. (27)) equal to $\left(f_{K} / f_{\pi}\right)^{2}$. It has been supplemented with nonet symmetry breaking for reasons already presented and with the success we saw.

The second mean is the BGP breaking [6] of the anomalous FKTUY Lagrangian illustrated by Eq. (18), which turns out to introduce a breaking matrix $X_{W}$ and a new breaking parameter $\ell_{W}$. Within the schemes presented up to now, the fit value found for this parameter (practically $\ell_{W}=1$ ) cancels out such a possibility, leaving us with only" nonet symmetry breaking and the fixed original BKY breaking scheme $\left(\ell_{A} \equiv\left(f_{K} / f_{\pi}\right)^{2}\right)$.

A third mean is however conceivable which has not yet been explored to our knowledge. One should note that the BKY breaking mechanism [11] implies a renormalization (or redefinition) of the pseudoscalar field matrix expressed through $X_{A}$; however, the $X_{V}$ breaking does not end up with a renormalization of the vector field matrix, which remains unchanged in the breaking procedure. In order to go on, let us postulate that the vector meson field matrix has also to be $\mathrm{SU}(3)$ broken, and that this can be done by performing the change :

$$
V \longrightarrow X_{T} V X_{T} \quad, \quad\left[X_{T}=\operatorname{diag}\left(1,1,1+\mathrm{c}_{\mathrm{T}}\right)\right]
$$

in Eq. (18), in complete analogy with the renormalization of the $P$ matrix. One could as well assume that this breaking affects directly the FKTUY Lagrangian, however one cannot avoid remarking that this looks indeed like a field renormalization, exactly as for $P$. Doing

\footnotetext{
${ }^{16}$ Of course, the mixing angles are to be fit, as seen above.
} 
this way, we have a priori 3 parameters at our disposal in order to describe the full set of data of relevance : $\ell_{A}, \ell_{W}$, and $\square \ell_{T}=\left(1+c_{T}\right)^{2}$.

The Lagrangian corresponding to this case is given in Eq. (A14). The coupling constants in Eqs. (A3) are unchanged in this new scheme except for $K^{*}$ 's which become :

$$
\left\{\begin{array}{l}
G_{K^{* 0} K^{0} \gamma}=-G \frac{\sqrt{K^{\prime}}}{3}\left(1+\ell_{W} \ell_{T}\right) \\
G_{K^{* \pm} K^{ \pm} \gamma}=G \frac{\sqrt{K^{\prime}}}{3}\left(2-\ell_{W} \ell_{T}\right)
\end{array}\right.
$$

where $K^{\prime}=\ell_{T} / \ell_{A}$. Eqs. (A4) and (A5) are also unchanged, provided one replaces there $Z$ by $Z^{\prime}=Z \ell_{T}^{2}$. Eqs. (A7) are, of course, unchanged $[8$.

Assuming no coupling to glue, we have performed the fit and found a perfect fit quality $\left(\chi^{2} / d o f=10.9 / 9\right)$; in this case, the $K^{* \pm}$ is found to contribute to the full $\chi^{2}$ for $1.6310^{-3}$, while the $K^{* 0}$ contribution increases with respect to previous modelling and becomes 0.68 , i.e. unsignificantly. The fit parameters common to the previous (no-glue) model are found unchanged (see second data column in Table 回), except for $\theta_{P}=-11.91^{\circ} \pm 1.10^{\circ}$, which thus has moved by only $0.3 \sigma$. This fit provides :

$$
\left\{\begin{array}{l}
\ell_{T}=1.24 \pm 0.06 \quad\left(c_{T}=0.113 \pm 0.025\right) \\
\ell_{W}=0.66 \pm 0.06 \quad\left(c_{W}=-0.340 \pm 0.058\right)
\end{array}\right.
$$

with a large correlation coefficient $\left(\ell_{T}, \ell_{W}\right)=-0.935$. This can easily be understood : the pseudoscalar mixing angle is practically fixed by the set of (single photon) radiative decays. Then, as shown indirectly by Fig. 1 in Ref. [13], a mixing angle of $\theta_{P} \simeq-11^{\circ}$ forces the value of $Z^{\prime}$ to be practically $1 / \ell_{A}$; thus, $\eta / \eta^{\prime}$ decays to two photons force $\ell_{T}$ and $\ell_{W}$ to fulfill $\ell_{W} \ell_{T}^{2} \simeq 1$.

Therefore, it is indeed possible to accommodate all decays we examine. However, there is an additional price to pay in order to include the $K^{* \pm}$ inside the fit set of data : we need to renormalize the vector field matrix (introduction of $\ell_{T}$ ) and simultaneously break the FKTUY Lagrangian à la BGP, in such way that these parameters fulfill an interesting condition

$$
\ell_{W} \ell_{T}^{2}=1
$$

Stated otherwise : accounting for this measurement implies to include one more parameter. However, this additional parameter plays in opposite directions for both $K^{*}$ radiative decays as illustrated by Eqs. (33). Assuming the validity of the condition proposed by Eq.

\footnotetext{
${ }^{17}$ Notice the square in the definition for $\ell_{T}$.

18 If $X_{T}$ actually follows from a renormalization of the vector field matrix, it might affect the expression for the leptonic decay widths considered. In this case, the values we get for $a$ and $\ell_{V}$ account effectively for this.
} 
(35), implies a highly non-trivial relation between the $K^{* \pm}$ and $K^{* 0}$ radiative decay widths (or coupling constants), which can hardly be accidental if it is fulfilled. The relation just above between $\ell_{W}$ and $\ell_{T}$ is however a numerical property and does not a priori mean that $X_{W}$ and $X_{T}$ are connected from some basic principle.

Therefore we have redone the fit described just above, requiring Eq. (35) among the corresponding fit parameters. We got a good fit quality $\left(\chi^{2} / d o f=11.07 / 10\right)$ with exactly the same parameter values as given in the second data column of Table $\mathbb{q}$ and additionally

$$
\ell_{T}=1.19 \pm 0.06 \quad, \quad\left(c_{T}=0.109 \pm 0.024\right)
$$

which turns to fix the BGP breaking parameter to $\ell_{W}=0.71 \pm 0.07$.

Thus, in order to account for $K^{*}$ radiative decays, two elementary breaking mechanisms interplay with algebraically related strengths. Even if somehow non-trivial, this procedure works without destroying the reconstruction quality of the $K^{* 0}$ radiative decay, which was not obvious, knowing that we were looking for a factor of 2 for one only of these two modes. The prediction of this last model for branching fractions are listed in Tables III and IV under the entry name " $K^{* \pm}$ Breaking". All predictions are clearly in nice agreement with all accepted data 30.

Nevertheless, this mechanism clearly complicates the full breaking picture which is otherwise quite simple. One can hope that new measurements for the $K^{* \pm}$ radiative decay will come soon from the CLEO, BaBar and BELLE detectors through the decay process $\tau^{ \pm} \rightarrow K^{* \pm} \nu_{\tau}$. This, anyway, would clarify the nature of the problem. If confirmed, this branching fraction would rise the question of wether $X_{T}$ can be a renormalization of the vector field matrix ; if the relation between $X_{T}$ and $X_{W}$ (the BGP breaking) is probably accidental, a connection between $X_{T}$ and $X_{V}$ (the second breaking matrix in the BKY breaking mechanism) is not excluded.

We do not discuss at length, the correlation between glue component and nonet symmetry, the conclusions reported above remain fully valid.

\section{CONCLUSION}

We have built a model aimed at describing the radiative and leptonic decays of light flavor mesons, including the two-photon decays of pseudoscalar mesons. This model relies heavily on the HLS model supplemented with the BKY breaking mechanism in order to account for $\mathrm{SU}(3)$ symmetry breaking. It has been shown that this is not enough to provide a satisfactory description of the available data, even by leaving free this breaking parameter and, even, by allowing for an additional breaking scheme (BGP).

A nice agreement is however reached by allowing, additionally, either nonet symmetry breaking in the pseudoscalar sector or having an additional singlet which affects essentially the $\eta^{\prime}$ meson. This has been named glue, but could be as well any additional kind of SU(3) singlet (a $c \bar{c}$ component for instance).

The picture that emerges from there is quite consistent and tends to indicate that present data do not require any breaking of the $S U(2)$ symmetry at a visible level in only radiative decays of light mesons.

We thus find that the radiative decays of the kind $V \rightarrow P \gamma$ and $P \rightarrow V \gamma$ allow one to predict quite precisely the decay widths for $P \rightarrow \gamma \gamma$. We also found, as expected from the 
BKY breaking mechanism, that the relation between the breaking parameter and $f_{K} / f_{\pi}$ is perfectly fit from data.

As a side result, we have shown that the HLS model in its anomalous sector leads to the traditional one angle mixing pattern for the $\left(\eta, \eta^{\prime}\right)$ system. In this case, the pseudoscalar mixing angle is $\theta_{P}=-11.59 \pm 0.76$ degrees in nice agreement with the value expected from linear mass formulae, but in poor agreement with ChPT expectations. This value for $\theta_{P}$ is, however, practically determined by only the radiative one-photon decays of light flavor mesons and therefore follows closely VMD expectations.

The single data which could require a special breaking procedure is the $K^{* \pm}$ radiative decay, if expected measurements confirm the present accepted data. The existing measurements can, however, be accommodated at the expense of complicating somehow the SU(3) breaking scheme in our VMD model. New measurements of this mode can be expected from $B$ factories in a near future ; this should tell definitely if such complications are really needed.

Finally, we have shown that effects due to nonet symmetry breaking and glue (and/or $c \bar{c}$ admixture) cannot be practically disentangled, and then, in order to guarantee presence or absence of glue inside light mesons, one has first to ascertain the level of nonet symmetry breaking.

\section{Acknowledgements}

We thank J.-M. Frère (ULB, Brussels) for reading the manuscript and providing useful comments. One of us (MB) acknowledges useful discussions with B. Moussalam (IPN, Orsay). HOC was supported by the US Department of Energy under contracts DE-FG0296ER40989 (UK) and DE-AC03-76SF00515 (SLAC). SE was supported by the Division des Affaires Internationales of IN2P3 and would like to thank the LPNHE Laboratory for its hospitality; VNI was supported by the Direction des Affaires Internationales of CNRS. Both SE and VNI are grateful to Eliane Perret (IN2P3) and Marcel Banner (LPNHE) for their help and support. 


\section{TABLES}

\begin{tabular}{|c|c|c|c|c|c|}
\hline \multirow[b]{2}{*}{$\begin{array}{c}\text { Breaking } \\
\text { Conditions }\end{array}$} & \multicolumn{3}{|c|}{$\begin{array}{c}\eta \rightarrow \gamma \gamma \\
\left(e^{+} e^{-} \rightarrow \eta e^{+} e^{-}\right)\end{array}$} & \multicolumn{2}{|c|}{$\begin{array}{c}\eta \rightarrow \gamma \gamma \\
\text { (Primakoff Effect) }\end{array}$} \\
\hline & $\begin{array}{l}\mathrm{SU}(3) \\
\text { only }\end{array}$ & $\begin{array}{c}\text { Nonet Symm. } \\
+\mathrm{SU}(3)\end{array}$ & $\begin{array}{c}\text { Glue } \\
+\mathrm{SU}(3)\end{array}$ & $\begin{array}{c}\text { Glue } \\
+\mathrm{SU}(3)\end{array}$ & $\begin{array}{c}\text { Nonet Symm. } \\
+\mathrm{SU}(3)\end{array}$ \\
\hline$G[\mathrm{GeV}]^{-1}$ & $0.703 \pm 0.002$ & $0.704 \pm 0.002$ & $0.704 \pm 0.002$ & $0.704 \pm 0.002$ & $0.704 \pm 0.002$ \\
\hline$x$ & 1. & $0.917 \pm 0.017$ & 1. & 1. & $0.854 \pm 0.025$ \\
\hline$\beta$ [deg.] & 0. & 0. & 0. & $21.34_{-4.06}^{+3.43}$ & 0. \\
\hline$\gamma$ [deg. $]$ & 0. & 0. & $26.38_{-2.79}^{+2.51}$ & $21.28_{-2.36}^{+2.30}$ & 0. \\
\hline$\ell_{A}$ & $1.41_{-0.41}^{+0.60}$ & 1.50 & 1.50 & 1.50 & $1.624 \pm 0.059$ \\
\hline$\ell_{W}$ & {$\left[1-0.2410^{-3}\right]_{-0.30}^{+0.42}$} & 1. & 1. & 1. & 1. \\
\hline$\ell_{V}$ & $1.376 \pm 0.031$ & $1.376 \pm 0.031$ & $1.376 \pm 0.031$ & $1.376 \pm 0.031$ & $1.374 \pm 0.031$ \\
\hline$a$ [HLS $]$ & $2.503 \pm 0.033$ & $2.502 \pm 0.034$ & $2.503 \pm 0.034$ & $2.503 \pm 0.034$ & $2.506 \pm 0.034$ \\
\hline$\theta_{V}[\mathrm{deg}]$. & $31.92 \pm 0.17$ & $31.92 \pm 0.17$ & $31.92 \pm 0.17$ & $31.90 \pm 0.17$ & $31.88 \pm 0.17$ \\
\hline$\theta_{P}[\mathrm{deg}]$. & $-13.94 \pm 0.94$ & $-11.59 \pm 0.76$ & $-10.67 \pm 0.79$ & $-6.04 \pm 1.36$ & $-5.53 \pm 2.08$ \\
\hline$\chi^{2} / d o f$ & $31.9 / 9$ & $10.9 / 10$ & $11.1 / 10$ & $6.3 / 9$ & $10.1 / 9$ \\
\hline
\end{tabular}

TABLE I. Fit results under various strategies. Parameter values written boldface means that they are not allowed to vary. Nonet Symmetry violation and glue in this table are exclusive of each other. $K^{*+} \rightarrow K^{+} \gamma$ is outside all fits; its distance to the value expected from fit is always about $5 \sigma$. 


\begin{tabular}{||c|c|c|c|c|c||}
\hline \hline \hline $\begin{array}{c}\text { Breaking } \\
\text { Conditions }\end{array}$ & $x=0.7$ & $x=0.9$ & $x=1$ & $x=1.1$ & $x=1.3$ \\
\hline \hline$\beta$ [deg. $]$ & $20.91_{-2.57}^{+2.30}$ & {$\left[-0.210^{-2}\right] \pm 9.09$} & $3.76_{-7.59}^{+6.04}$ & $5.55_{-6.81}^{+5.22}$ & $7.45_{-5.76}^{+4.50}$ \\
\hline$\gamma[$ deg. $]$ & $-18.70 \pm 3.67$ & {$\left[-.5610^{-4}\right] \pm 11.87$} & $23.67_{-4.87}^{+6.23}$ & $33.51_{-3.67}^{+4.78}$ & $44.95_{-2.64}^{+3.32}$ \\
\hline \hline$\theta_{P}[$ deg. $]$ & $-8.75 \pm 1.01$ & $-11.33 \pm 00.73$ & $-10.07 \pm 0.79$ & $-9.70 \pm 0.72$ & $-8.23 \pm 0.61$ \\
\hline \hline$\chi^{2} /$ dof & $148.5 / 9$ & $11.9 / 9$ & $10.9 / 9$ & $10.9 / 9$ & $10.9 / 9$ \\
\hline \hline \hline
\end{tabular}

TABLE II. Main fit results fixing the nonet symmetry violation parameter $x$ to various fixed values. The BKY parameter is fixed to standard $\mathrm{SU}(3)$ breaking $\ell_{A}=1.5$, the BGP parameter is fixed to its no-breaking value $\ell_{A}=1.0$ and the process $K^{*+} \rightarrow K^{+} \gamma$ is outside all fits. Parameter values written boldface, means that they are not allowed to vary. 


\begin{tabular}{|c|c|c|c|c|}
\hline Process & $\begin{array}{l}\text { Nonet Sym. } \\
\quad+\mathrm{SU}(3)\end{array}$ & $\begin{array}{c}\text { Glue } \\
+\mathrm{SU}(3)\end{array}$ & $\begin{array}{c}K^{* \pm} \\
\text { Breaking }\end{array}$ & PDG \\
\hline$\rho \rightarrow \pi^{0} \gamma\left(\times 10^{4}\right)$ & $5.16 \pm 0.03$ & $5.16 \pm 0.03$ & $5.16 \pm 0.03$ & $6.8 \pm 1.7$ \\
\hline$\rho \rightarrow \pi^{ \pm} \gamma\left(\times 10^{4}\right)$ & $5.12 \pm 0.03$ & $5.12 \pm 0.03$ & $5.12 \pm 0.03$ & $4.5 \pm 0.5$ \\
\hline$\rho \rightarrow \eta \gamma\left(\times 10^{4}\right)$ & $3.25 \pm 0.10$ & $3.28 \pm 0.10$ & $3.31 \pm 0.09$ & $2.4_{-0.9}^{+0.8}$ \\
\hline$\eta^{\prime} \rightarrow \rho \gamma\left(\times 10^{2}\right)$ & $33.1 \pm 2.0$ & $33.7 \pm 2.0$ & $33.0 \pm 1.8$ & $30.2 \pm 1.3$ \\
\hline$K^{* \pm} \rightarrow K^{ \pm} \gamma\left(\times 10^{4}\right)$ & $5.66 \pm 0.03$ & $5.66 \pm 0.03$ & $9.80 \pm 0.93$ & $9.9 \pm 0.9$ \\
\hline$K^{* 0} \rightarrow K^{0} \gamma\left(\times 10^{3}\right)$ & $2.30 \pm 0.01$ & $2.30 \pm 0.01$ & $2.32 \pm 0.02$ & $2.3 \pm 0.2$ \\
\hline$\omega \rightarrow \pi^{0} \gamma\left(\times 10^{2}\right)$ & $8.50 \pm 0.05$ & $8.50 \pm 0.05$ & $8.50 \pm 0.05$ & $8.5 \pm 0.5$ \\
\hline$\omega \rightarrow \eta \gamma\left(\times 10^{4}\right)$ & $8.0 \pm 0.2$ & $8.1 \pm 0.2$ & $8.12 \pm 0.19$ & $6.5 \pm 1.0$ \\
\hline$\eta^{\prime} \rightarrow \omega \gamma\left(\times 10^{2}\right)$ & $2.8 \pm 0.2$ & $2.9 \pm 0.2$ & $2.8 \pm 0.2$ & $3.01 \pm 0.30$ \\
\hline$\phi \rightarrow \pi^{0} \gamma\left(\times 10^{3}\right)$ & $1.27 \pm 0.13$ & $1.28 \pm 0.12$ & $1.26 \pm 0.13$ & $1.31 \pm 0.13$ \\
\hline$\phi \rightarrow \eta \gamma\left(\times 10^{2}\right)$ & $1.25 \pm 0.04$ & $1.25 \pm 0.05$ & $1.22 \pm 0.04$ & $1.26 \pm 0.06$ \\
\hline$\phi \rightarrow \eta^{\prime} \gamma\left(\times 10^{4}\right)$ & $0.61 \pm 0.027$ & $0.55 \pm 0.03$ & $0.63 \pm 0.02$ & $1.2_{-0.5}^{+0.7}$ \\
\hline$\eta \rightarrow \gamma \gamma\left(\times 10^{2}\right)$ & $40.5 \pm 1.7$ & $40.8 \pm 1.8$ & $41.5 \pm 1.4$ & $39.21 \pm 0.34$ \\
\hline$\eta^{\prime} \rightarrow \gamma \gamma\left(\times 10^{2}\right)$ & $2.1 \pm 0.1$ & $2.1 \pm 0.1$ & $2.1 \pm 0.1$ & $2.11 \pm 0.13$ \\
\hline
\end{tabular}

TABLE III. Branching fractions from fits for radiative decays under various conditions of symmetry breakings. Note that the rate for $K^{* \pm}$ is a prediction in the first two data columns, while the corresponding data is included in the fit which leads to the third data column. 


\begin{tabular}{|c|c|c|c|c|}
\hline Process & $\begin{array}{c}\text { Nonet Sym. } \\
+\mathrm{SU}(3)\end{array}$ & $\begin{array}{c}\text { Glue } \\
+\mathrm{SU}(3)\end{array}$ & $\begin{array}{c}K^{* \pm} \\
\text { Breaking }\end{array}$ & $\overline{\mathrm{PDG}}$ \\
\hline$\rho \rightarrow e^{+} e^{-}\left(\times 10^{5}\right)$ & $4.6 \pm 0.1$ & $4.6 \pm 0.1$ & $4.6 \pm 0.1$ & $4.49 \pm 0.22$ \\
\hline$\omega \rightarrow e^{+} e^{-}\left(\times 10^{5}\right)$ & $7.0 \pm 0.2$ & $7.0 \pm 0.2$ & $7.0 \pm 0.2$ & $7.07 \pm 0.19$ \\
\hline$\phi \rightarrow e^{+} e^{-}\left(\times 10^{4}\right)$ & $3.10 \pm 0.16$ & $3.11 \pm 0.16$ & $3.08 \pm 0.16$ & $2.99 \pm 0.08$ \\
\hline$\phi \rightarrow K^{ \pm} K^{\mp}\left(\times 10^{2}\right)$ & $52.3 \pm 2.8$ & $52.3 \pm 2.7$ & $52.3 \pm 2.8$ & $49.1 \pm 0.8$ \\
\hline$\phi \rightarrow K^{0} \bar{K}^{0}\left(\times 10^{2}\right)$ & $34.0 \pm 1.7$ & $34.0 \pm 1.7$ & $34.1 \pm 1.7$ & $34.1 \pm 0.6$ \\
\hline
\end{tabular}

TABLE IV. Branching fractions from fits under various conditions of symmetry breakings. Note that hadronic branching fractions for $\phi$ are predictions and that the corresponding experimental value do not play any role in these predictions. 


\section{APPENDIX A:}

\section{The Standard Broken $V V P$ Lagrangian}

Expressed in terms of $\ell_{A}$ and $\ell_{W}$ defined in the body of the text, the $\mathrm{SU}(3)$ broken FKTUY Lagrangian of relevance for us :

$$
\mathcal{L}=-\frac{3 g^{2}}{4 \pi^{2} f_{\pi}} \epsilon^{\mu \nu \alpha \beta} \operatorname{Tr}\left[\partial_{\mu} V_{\nu} X_{W} \partial_{\alpha} V_{\beta} X_{A}^{-1 / 2} P^{\prime} X_{A}^{-1 / 2}\right]=C \epsilon^{\mu \nu \alpha \beta} F_{\mu \nu \alpha \beta}
$$

can be developed in the following way :

$$
\begin{aligned}
F_{\mu \nu \alpha \beta}=\partial_{\mu} \rho_{\nu}^{0} & {\left[\frac{1}{2 \sqrt{\ell_{A}}}\left(\partial_{\alpha} K_{\beta}^{*-} K^{+}+\partial_{\alpha} K_{\beta}^{*+} K^{-}-\partial_{\alpha} \bar{K}_{\beta}^{* 0} K^{0}-\partial_{\alpha} K_{\beta}^{* 0} \bar{K}^{0}\right)\right.} \\
& \left.+\partial_{\alpha} \omega_{\beta} \pi^{0}+\partial_{\alpha} \rho_{\beta}^{0}\left(\frac{\pi^{8}}{2 \sqrt{3}}+\frac{x \eta_{0}}{\sqrt{6}}\right)\right] \\
+ & \partial_{\mu} \omega_{\nu}\left[\frac{1}{2 \sqrt{\ell_{A}}}\left(\partial_{\alpha} K_{\beta}^{*-} K^{+}+\partial_{\alpha} K_{\beta}^{*+} K^{-}+\partial_{\alpha} \bar{K}_{\beta}^{* 0} K^{0}+\partial_{\alpha} K_{\beta}^{* 0} \bar{K}^{0}\right)\right. \\
& \left.+\left(\partial_{\alpha} \rho_{\beta}^{+} \pi^{-}+\partial_{\alpha} \rho_{\beta}^{-} \pi^{+}\right)+\partial_{\alpha} \omega_{\beta}\left(\frac{\pi^{8}}{2 \sqrt{3}}+\frac{x \eta_{0}}{\sqrt{6}}\right)\right] \\
+ & \partial_{\mu} \phi_{\nu}\left[\frac{\ell_{W}}{\sqrt{2 \ell_{A}}}\left(\partial_{\alpha} K_{\beta}^{*-} K^{+}+\partial_{\alpha} K_{\beta}^{*+} K^{-}+\partial_{\alpha} \bar{K}_{\beta}^{* 0} K^{0}+\partial_{\alpha} K_{\beta}^{* 0} \bar{K}^{0}\right)\right. \\
& \left.+\frac{\ell_{W}}{\ell_{A}} \partial_{\alpha} \phi_{\beta}\left(-\frac{1}{\sqrt{3}} \pi^{8}+\frac{1}{\sqrt{6}} x \eta_{0}\right)\right] \\
+ & \frac{1}{\sqrt{2 \ell_{A}}}\left[\partial_{\mu} \rho_{\nu}^{+}\left(\partial_{\alpha} K_{\beta}^{* 0} K^{-}+\partial_{\alpha} K_{\beta}^{*-} K^{0}\right)+\partial_{\mu} \rho_{\nu}^{-}\left(\partial_{\alpha} \bar{K}_{\beta}^{* 0} K^{+}+\partial_{\alpha} K_{\beta}^{*+} \bar{K}^{0}\right)\right] \\
+ & 2 \partial_{\mu} \rho_{\nu}^{+} \partial_{\alpha} \rho_{\beta}^{-}\left(\frac{\pi^{8}}{2 \sqrt{3}}+\frac{x \eta_{0}}{\sqrt{6}}\right) \\
+ & \frac{\ell_{W}}{\sqrt{2}}\left(\partial_{\mu} K_{\nu}^{*-} \partial_{\alpha} K_{\beta}^{* 0} \pi^{+}+\partial_{\mu} K_{\nu}^{*+} \partial_{\alpha} \bar{K}_{\beta}^{* 0} \pi^{-}\right) \\
+ & \partial_{\mu} K_{\nu}^{*-} \partial_{\alpha} K_{\beta}^{*+}\left[\frac{1}{2} \pi^{0}-\frac{2-R}{2 R \sqrt{3}} \pi^{8}+\frac{1+R}{R \sqrt{6}} x \eta_{0}\right] \\
+ & \partial_{\mu} \bar{K}_{\nu}^{* 0} \partial_{\alpha} K_{\beta}^{* 0}\left[-\frac{1}{2} \pi^{0}-\frac{2-R}{2 R \sqrt{3}} \pi^{8}+\frac{1+R}{R \sqrt{6}} x \eta_{0}\right] .
\end{aligned}
$$

where $R=\ell_{A} \ell_{W}$ and $C=-3 g^{2} /\left(8 \pi^{2} f_{\pi}\right)$. The expression for the functional $F$ in terms of the physical fields $\omega, \phi, \eta$ and $\eta^{\prime}$ can be trivially deduced.

\section{Matrix Elements with SU(3) and Nonet Broken Symmetries}

In terms of the angles $\left(\delta_{V}, \delta_{A}\right)$ of physical states with respect to ideal mixing, as

defined in Section IV, the coupling constants at vertices $V P \gamma$ which can be deduced from the Lagrangian in Eq. (A2) are : 


$$
\begin{cases}G_{\rho^{0} \pi^{0} \gamma}= & \frac{1}{3} G \\ G_{\rho^{ \pm} \pi^{ \pm} \gamma}= & \frac{1}{3} G \\ G_{K^{* 0} K^{0} \gamma}= & -G \frac{\sqrt{K}}{3}\left(1+\ell_{W}\right) \\ G_{K^{* \pm} K^{ \pm} \gamma}= & G \frac{\sqrt{K}}{3}\left(2-\ell_{W}\right) \\ G_{\rho^{0} \eta \gamma}= & \frac{1}{3} G\left[\sqrt{2}(1-x) \cos \delta_{P}-(2 x+1) \sin \delta_{P}\right] \\ G_{\rho^{0} \eta^{\prime} \gamma}= & \frac{1}{3} G\left[\sqrt{2}(1-x) \sin \delta_{P}+(2 x+1) \cos \delta_{P}\right] \\ G_{\omega \pi^{0} \gamma}= & G \cos \delta_{V} \\ G_{\phi \pi^{0} \gamma}= & -G \sin \delta_{V}\end{cases}
$$

and :

$$
\left\{\begin{aligned}
G_{\omega \eta \gamma}= & \frac{1}{9} G\left[-(2 x+1) \cos \delta_{V} \sin \delta_{P}-2 Z(2+x) \sin \delta_{V} \cos \delta_{P}\right. \\
& \left.+2 \sqrt{2} Z(1-x) \sin \delta_{V} \sin \delta_{P}+\sqrt{2}(1-x) \cos \delta_{V} \cos \delta_{P}\right] \\
G_{\omega \eta^{\prime} \gamma}= & \frac{1}{9} G\left[(2 x+1) \cos \delta_{V} \cos \delta_{P}-2 Z(2+x) \sin \delta_{V} \sin \delta_{P}\right. \\
& \left.-2 \sqrt{2} Z(1-x) \sin \delta_{V} \cos \delta_{P}+\sqrt{2}(1-x) \cos \delta_{V} \sin \delta_{P}\right] \\
G_{\phi \eta \gamma}=\quad & \frac{1}{9} G\left[(2 x+1) \sin \delta_{V} \sin \delta_{P}-2 Z(2+x) \cos \delta_{V} \cos \delta_{P}\right. \\
& \left.+2 \sqrt{2} Z(1-x) \cos \delta_{V} \sin \delta_{P}-\sqrt{2}(1-x) \sin \delta_{V} \cos \delta_{P}\right] \\
G_{\phi \eta^{\prime} \gamma}=- & \frac{1}{9} G\left[(2 x+1) \sin \delta_{V} \cos \delta_{P}+2 Z(2+x) \cos \delta_{V} \sin \delta_{P}\right. \\
& \left.+2 \sqrt{2} Z(1-x) \cos \delta_{V} \cos \delta_{P}+\sqrt{2}(1-x) \sin \delta_{V} \sin \delta_{P}\right]
\end{aligned}\right.
$$

where the breaking parameters come through the combinations $K=1 / \ell_{A}$ and $Z=\ell_{W} / \ell_{A}$. The dependence upon the nonet symmetry breaking parameter $x$ is explicit. The basic parameter $G$ yields the following expression :

$$
G=\frac{C e}{2 g}=-\frac{3 e g}{8 \pi^{2} f_{\pi}}
$$

which depends on the vector meson universal coupling $g$ and the pion decay constant $f_{\pi}$. We will fit the absolute value of $G$.

Correspondingly the matrix elements for the decays $\pi^{0} / \eta / \eta^{\prime} \rightarrow \gamma \gamma$ are :

$$
\left\{\begin{aligned}
G_{\eta \gamma \gamma} & =-\frac{\alpha_{e m}}{\pi \sqrt{3} f_{\pi}}\left[\frac{5-2 Z}{3} \cos \theta_{P}-\sqrt{2} \frac{5+Z}{3} x \sin \theta_{P}\right] \\
G_{\eta^{\prime} \gamma \gamma} & =-\frac{\alpha_{e m}}{\pi \sqrt{3} f_{\pi}}\left[\frac{5-2 Z}{3} \sin \theta_{P}+\sqrt{2} \frac{5+Z}{3} x \cos \theta_{P}\right] \\
G_{\pi^{0} \gamma \gamma} & =-\frac{\alpha_{e m}}{\pi f_{\pi}}
\end{aligned}\right.
$$


Finally the $V-\gamma$ couplings which enter Eq. (15) are constant within this framework and are :

$$
\left\{\begin{array}{l}
f_{\rho \gamma}=a f_{\pi}^{2} g \\
f_{\omega \gamma}=\frac{f_{\rho \gamma}}{3}\left[\cos \delta_{V}+\ell_{V} \sqrt{2} \sin \delta_{V}\right] \\
f_{\phi \gamma}=-\frac{f_{\rho \gamma}}{3}\left[\sin \delta_{V}-\ell_{V} \sqrt{2} \cos \delta_{V}\right]
\end{array}\right.
$$

We have defined above $K$ and $Z$ in terms of the breaking parameters of Bando Kugo Yamawaki (BKY) [11], and of that of Bramon, Grau and Pancheri (BGP) [6] :

$$
\ell_{A}=1+c_{A} \quad, \quad \ell_{W}=1+c_{W} \quad \ell_{V}=\left(1+c_{V}\right)^{2} .
$$

\section{Matrix Elements with All Breakings and Coupling to Glue}

By inverting Eq. (2), we get expressions for $\pi_{8}$ and $\eta_{0}$ in terms of the three mixing angles

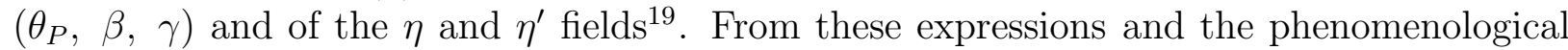
Lagrangian of Eq. (A2), we can recompute the expressions corresponding to Eqs. (A3), (A4) and (A6).

Of course, all $G_{V P \gamma}$ 's, where $P$ is neither one of $\eta$ and $\eta^{\prime}$, are unchanged and coincide with the corresponding expressions given in Eqs. (A3). The other expressions become :

$$
\left\{\begin{aligned}
G_{\rho^{0} \eta \gamma}= & \frac{\cos \beta}{3} G\left\{\sqrt{2}(1-x) \cos \delta_{P}-(2 x+1) \sin \delta_{P}\right\} \\
G_{\rho^{0} \eta^{\prime} \gamma}= & \frac{\cos \gamma}{3} G\left\{[\sqrt{2}(1-x)+(2 x+1) \sin \beta \tan \gamma] \tan \delta_{P}\right. \\
& \left.+[(2 x+1)-\sqrt{2}(1-x) \sin \beta \tan \gamma] \cos \delta_{P}\right\}
\end{aligned}\right.
$$

\footnotetext{
${ }^{19}$ We ignore the spurious $\eta^{\prime \prime}$ as long as we don’t know what particle it could correspond to.
} 


$$
\left\{\begin{aligned}
G_{\omega \eta \gamma}= & \frac{\cos \beta}{9} G\left\{-(2 x+1) \cos \delta_{V} \sin \delta_{P}-2 Z(2+x) \sin \delta_{V} \cos \delta_{P}\right. \\
& \left.+2 \sqrt{2} Z(1-x) \sin \delta_{V} \sin \delta_{P}+\sqrt{2}(1-x) \cos \delta_{V} \cos \delta_{P}\right\} \\
G_{\omega \eta^{\prime} \gamma}=\quad & \frac{\cos \gamma}{9} G\left\{[(2 x+1)-\sqrt{2}(1-x) \sin \beta \tan \gamma] \cos \delta_{V} \cos \delta_{P}\right. \\
& -2 Z[(2+x)+\sqrt{2}(1-x) \sin \beta \tan \gamma] \sin \delta_{V} \sin \delta_{P} \\
& -2 Z[\sqrt{2}(1-x)-(2+x) \sin \beta \tan \gamma)] \sin \delta_{V} \cos \delta_{P} \\
& \left.+[\sqrt{2}(1-x)+(2 x+1) \sin \beta \tan \gamma] \cos \delta_{V} \sin \delta_{P}\right\} \\
& \frac{\cos \beta}{9} G\left\{(2 x+1) \sin \delta_{V} \sin \delta_{P}-2 Z(2+x) \cos \delta_{V} \cos \delta_{P}\right. \\
& \left.+2 \sqrt{2} Z(1-x) \cos \delta_{V} \sin \delta_{P}-\sqrt{2}(1-x) \sin \delta_{V} \cos \delta_{P}\right\} \\
G_{\phi \eta \gamma}= & \frac{\cos \gamma}{9} G\left\{[(2 x+1)-\sqrt{2}(1-x) \sin \beta \tan \gamma] \sin \delta_{V} \cos \delta_{P}\right. \\
& +2 Z[(2+x)+\sqrt{2}(1-x) \sin \beta \tan \gamma] \cos \delta_{V} \sin \delta_{P} \\
& +2 Z[\sqrt{2}(1-x)-(2+x) \sin \beta \tan \gamma] \cos \delta_{V} \cos \delta_{P} \\
G_{\phi \eta^{\prime} \gamma}=- & \left.+\sqrt{2}(1-x)+(2 x+1) \sin \beta \tan \gamma] \sin \delta_{V} \sin \delta_{P}\right\}
\end{aligned}\right.
$$

Correspondingly the matrix element for the decay $\pi^{0} \rightarrow \gamma \gamma$ is not affected, while the amplitudes for $\eta / \eta^{\prime} \rightarrow \gamma \gamma$ become :

$$
\left\{\begin{aligned}
G_{\eta \gamma \gamma}= & -\frac{\alpha_{e m} \cos \beta}{\pi \sqrt{3} f_{\pi}}\left\{\frac{5-2 Z}{3} \cos \theta_{P}-\sqrt{2} \frac{5+Z}{3} x \sin \theta_{P}\right\} \\
G_{\eta^{\prime} \gamma \gamma}= & -\frac{\alpha_{e m} \cos \gamma}{\pi \sqrt{3} f_{\pi}}\left\{\left[\frac{5-2 Z}{3}+\sqrt{2} \frac{5+Z}{3} x \sin \beta \tan \gamma\right] \sin \theta_{P}\right. \\
& \left.+\left[\sqrt{2} \frac{5+Z}{3} x-\frac{5-2 Z}{3} \sin \beta \tan \gamma\right] \cos \theta_{P}\right\}
\end{aligned}\right.
$$

\section{The $K^{*}$ Broken $V V P$ Lagrangian}

In order to describe fully the $K^{*}$ sector of light meson radiative decays, we propose to use the Lagrangian given in Eq. (A1), supplemented with the replacement $V \rightarrow X_{T} V X_{T}$, with

$$
X_{T}=\operatorname{diag}\left(1,1,1+\mathrm{c}_{\mathrm{T}}\right)
$$

where the breaking parameter, expected to be small, has been denoted $c_{T}$. In the following we use the definition

$$
\ell_{T}=\left(1+c_{T}\right)^{2}
$$

together with the breaking parameters already defined by Eqs. (A8) and the nonet symmetry breaking factor $x$. 
The expanded expression for the FKTUY Lagrangian broken in this way is still given by Eq. (A1) with

$$
\begin{aligned}
F_{\mu \nu \alpha \beta}=\partial_{\mu} \rho_{\nu}^{0}[ & {\left[\frac{1}{2} \sqrt{\frac{\ell_{T}}{\ell_{A}}}\left(\partial_{\alpha} K_{\beta}^{*-} K^{+}+\partial_{\alpha} K_{\beta}^{*+} K^{-}-\partial_{\alpha} \bar{K}_{\beta}^{* 0} K^{0}-\partial_{\alpha} K_{\beta}^{* 0} \bar{K}^{0}\right)\right.} \\
& \left.+\partial_{\alpha} \omega_{\beta} \pi^{0}+\partial_{\alpha} \rho_{\beta}^{0}\left(\frac{\pi^{8}}{2 \sqrt{3}}+\frac{x \eta_{0}}{\sqrt{6}}\right)\right] \\
+ & \partial_{\mu} \omega_{\nu}\left[\frac{1}{2} \sqrt{\frac{\ell_{T}}{\ell_{A}}}\left(\partial_{\alpha} K_{\beta}^{*-} K^{+}+\partial_{\alpha} K_{\beta}^{*+} K^{-}+\partial_{\alpha} \bar{K}_{\beta}^{* 0} K^{0}+\partial_{\alpha} K_{\beta}^{* 0} \bar{K}^{0}\right)\right. \\
& \left.+\left(\partial_{\alpha} \rho_{\beta}^{+} \pi^{-}+\partial_{\alpha} \rho_{\beta}^{-} \pi^{+}\right)+\partial_{\alpha} \omega_{\beta}\left(\frac{\pi^{8}}{2 \sqrt{3}}+\frac{x \eta_{0}}{\sqrt{6}}\right)\right] \\
+ & \partial_{\mu} \phi_{\nu}\left[\ell_{W} \sqrt{\frac{\ell_{T}^{3}}{2 \ell_{A}}}\left(\partial_{\alpha} K_{\beta}^{*-} K^{+}+\partial_{\alpha} K_{\beta}^{*+} K^{-}+\partial_{\alpha} \bar{K}_{\beta}^{* 0} K^{0}+\partial_{\alpha} K_{\beta}^{* 0} \bar{K}^{0}\right)\right. \\
& \left.+\frac{\ell_{W} \ell_{T}^{2}}{\ell_{A}} \partial_{\alpha} \phi_{\beta}\left(-\frac{1}{\sqrt{3}} \pi^{8}+\frac{1}{\sqrt{6}} x \eta_{0}\right)\right] \\
+ & \sqrt{\frac{\ell_{T}}{2 \ell_{A}}}\left[\partial_{\mu} \rho_{\nu}^{+}\left(\partial_{\alpha} K_{\beta}^{* 0} K^{-}+\partial_{\alpha} K_{\beta}^{*-} K^{0}\right)+\partial_{\mu} \rho_{\nu}^{-}\left(\partial_{\alpha} \bar{K}_{\beta}^{* 0} K^{+}+\partial_{\alpha} K_{\beta}^{*+} \bar{K}^{0}\right)\right] \\
+ & 2 \partial_{\mu} \rho_{\nu}^{+} \partial_{\alpha} \rho_{\beta}^{-}\left(\frac{\pi^{8}}{2 \sqrt{3}}+\frac{x \eta_{0}}{\sqrt{6}}\right) \\
+ & \frac{\ell_{W} \ell_{T}}{\sqrt{2}}\left(\partial_{\mu} K_{\nu}^{*-} \partial_{\alpha} K_{\beta}^{* 0} \pi^{+}+\partial_{\mu} K_{\nu}^{*+} \partial_{\alpha} \bar{K}_{\beta}^{* 0} \pi^{-}\right) \\
+ & \ell_{T} \partial_{\mu} K_{\nu}^{*-} \partial_{\alpha} K_{\beta}^{*+}\left[\frac{1}{2} \pi^{0}-\frac{2-R}{2 R \sqrt{3}} \pi^{8}+\frac{1+R}{R \sqrt{6}} x \eta_{0}\right] \\
+ & \ell_{T} \partial_{\mu} \bar{K}_{\nu}^{* 0} \partial_{\alpha} K_{\beta}^{* 0}\left[-\frac{1}{2} \pi^{0}-\frac{2-R}{2 R \sqrt{3}} \pi^{8}+\frac{1+R}{R \sqrt{6}} x \eta_{0}\right] .
\end{aligned}
$$

where $R=\ell_{A} \ell_{W}$, as in Eq. (A2). 


\section{REFERENCES}

[1] J. Bartelski and S. Tatur, Phys. Lett. B289 (1992) 429.

[2] G. Morpurgo, Phys. Rev. D42 (1990) 1497 ; Phys. Rev. D46 (1992) 4068 ; G. Dillon and G. Morpurgo, Phys. Rev. D53 (1995) 3754.

[3] S.I. Dolinsky el al., Phys. Rep. 202 (1991) 99.

[4] P. O'Donnell, Rev. of Mod. Phys. 53 (1981) 673.

[5] M. Benayoun, Ph. Leruste, L. Montanet and J.-L. Narjoux, Zeit. Phys. C65 (1995) 399.

[6] A. Bramon, A. Grau and G. Pancheri, Phys. Lett. B344, 240 (1995).

[7] M. Hashimoto, Phys. Lett. B381, 465 (1996); Phys. Rev. D 54, 5611 (1996).

[8] A. Bramon, R. Escribano and M.D. Scadron, Eur. Phys. J. C7, 271 (1997) hepph/9711229.

[9] M. Bando, T. Kugo, S. Uehara, K. Yamawaki and T. Yanagida, Phys. Rev. Lett. 54, 1215 (1985); M. Bando, T. Kugo and K. Yamawaki, Phys. Rep. 164, 217 (1985).

[10] T. Fujiwara, T. Kugo, H. Terao, S. Uehara and K. Yamawaki, Prog. Th. Phys. 73, 926 (1985).

[11] M. Bando, T. Kugo and K. Yamawaki, Nucl. Phys. B259, 493 (1985).

[12] M. Benayoun and H.B. O'Connell, Phys. Rev. D58 (1998) 074006.

[13] P. Ball, J.-M. Frère and M. Tytgat, Phys. Lett. B365 (1996) 367.

[14] M. Benayoun et al., Zeit. Phys. C58 (1993) 31.

[15] M.S. Chanowitz, Phys. Rev. Lett. 35 (1975) 977 ; Phys. Rev. Lett. 44 (1980) 59.

[16] J. F. Donoghue, B. R. Holstein and Y. R. Lin, Phys. Rev. Lett. 55 (1985) 2766.

[17] F. J. Gilman and R. Kauffman, Phys. Rev. D36 (1987) 2761.

[18] G.M. Shore and G. Veneziano, Nucl. Phys. B381 (1992) 3.

[19] J. S. Bell and R. Jackiew, Nuov. Cim. 60A (1969) 47 ; S. L. Adler, Phys. Rev. 177 (1969) 2426.

[20] J. Wess and B. Zumino, Phys. Lett. 37B (1971) 95.

[21] E. Witten, Nucl. Phys. B223 (1983) 422.

[22] H. Leutwyler, Nucl. Phys. Proc. Suppl. 64, 223 (1998) hep-ph/9709408.

[23] R. Kaiser and H. Leutwyler, hep-ph/9806336.

[24] T. Feldman and P. Kroll, Eur. Phys. J. C5 (1998) 327 ; Phys. Rev. D58 (1998) 057501.

[25] T. Feldman, P. Kroll and B.Stech, hep-ph/9812269, Dec. 1998.

[26] R. Escribano and J.M. Frere, hep-ph/9901405.

[27] K. Maltman, Phys. Lett. B351, 56 (1995) hep-ph/9504252; K. Maltman and T. Goldman, Nucl. Phys. A572, 682 (1994); K. Maltman, Phys. Lett. B313, 203 (1993).

[28] K. Maltman, H.B. O'Connell and A.G. Williams, Phys. Lett. B376, 19 (1996) hepph/9601309; H.B. O'Connell, A.W. Thomas and A.G. Williams, Nucl. Phys. A623, 559 (1997) hep-ph/9703248; K. Maltman, Phys. Lett. B362, 11 (1995) nucl-th/9506024.

[29] H.B. O'Connell, B.C. Pearce, A.W. Thomas and A.G. Williams, Phys. Lett. B336, 1 (1994) hep-ph/9405273; S. Coleman and H.J. Schnitzer, Phys. Rev. 134, B863 (1964).

[30] C. Caso et al. Eur. Phys. Journ. C3 (1998) 1.

[31] M. Benayoun, S.I. Eidelman and V.N. Ivanchenko, Zeit. Phys. C72 (1996) 221.

[32] R.R. Akhmetshin et al., Phys. Lett. B364 (1995) 199.

[33] M.N. Achasov et al., JETP Lett. 68 (1998) 573.

[34] R.R. Akhmetshin et al., Phys. Lett. B415 (1997) 445. 
[35] V. M. Aulchenko et al., Phys. Lett. B436 (1998) 199 ; M.N. Achasov et al., Preprint Budker INP 98-65, Novosibirsk, 1998.

[36] V.N. Ivanchenko, "Review of Radiative decays Study", in Proceedings of the Workshop on Physics and Detectors for DAPHNE '95, Frascati, April 4-7, 1995, R. Baldini et al. Edts, INFN-Laboratory Nazionali di Frascati, Frascati, 1995.

[37] A. Bramon and M. Greco, Il Nuov. Cim. 14A (1973) 323 ; Phys. Lett. B48 (1974) 137.

[38] M. Harada and J. Schechter, Phys. Rev. D54, 3394 (1996).

[39] F. Klingl, N. Kaiser and W. Weise, Zeit. Phys. A356 (1996) 193.

[40] G. Grunberg, Phys. Lett. B388 (1996) 173.

[41] B.H. Behrens, Phys. Rev. Lett. 80 (1998) 3710.

[42] A.A. Petrov, Phys. Rev. D58 (1998) 054004.

[43] P. Minkowski and W. Ochs, hep-ph/9811518.

[44] C. Amsler, Rev. of Mod. Phys. 70 (1998) 1293.

[45] H.B. O'Connell, B.C. Pearce, A.W. Thomas and A.G. Williams, Prog. Part. Nucl. Phys. 39 (1996) 201.

[46] M. Benayoun et al., Eur. Phys. J. C2 (1998) 269.

[47] R.R. Akhmetshin et al., "Measurement of the $e^{+} e^{-} \rightarrow \pi^{+} \pi^{-}$cross-section with the CMD-2 detector," presented by I.B.Logashenko, Proc. of the VII Int. Conf. On Hadron Spectroscopy (Hadron'97), BNL, Upton NY, August 1997, p.787-792, S.U.Chung and H.J.Willutzki Editors, AIP Conference Proceedings 432, Woodbury, New York.

[48] P. Jain et al., Phys. Rev. D37 (1988) 3252.

[49] O. Hajuj, Zeit. Phys. C60 (1993) 357.

[50] H. Leutwyler, Phys. Lett. B374 (1996) 181.

[51] S. Gardner and H.B. O'Connell, Phys. Rev. D59, 076002 (1999) hep-ph/9809224.

[52] M. Takizawa, Y. Nemoto and M. Oka, Phys. Rev. D55 (1997) 4083.

[53] L.M. Barkov et al., Nucl. Phys. B256 (1985) 365.

[54] R.R. Akhmetshin et al., "Measurement of $e^{+} e^{-} \rightarrow \pi^{+} \pi^{-}$cross section around the $\rho$ meson", hep-ex/9904027.

[55] A. Bramon and M.D. Scadron, Phys. Lett. B234 (1990) 346.

[56] A. Bramon, R. Escribano and M.D. Scadron, Phys. Lett. B403 (1997) 339.

[57] G. Dillon and G. Morpurgo, Zeit. Phys. C64 (1994) 467.

[58] J. Gasser and H. Leutwyler, Nucl. Phys. B250 (1985) 465; Nucl. Phys. B250 (1985) 517.

[59] A. Bramon, A. Grau and G. Pancheri, Phys. Lett. B345, 263 (1995) hep-ph/9411269.

[60] A. Abele et al., Phys. Lett. B402 (1997) 195.

[61] M. Benayoun, H.B. O'Connell and A.G. Williams, Phys. Rev. D59, 074020 (1999) hep-ph/9807537. 